\title{
The Formation of Nonzonal Jets over Sloped Topography
}

\author{
EMMA J. D. BOLAND \\ The University of Cambridge, and The British Antarctic Survey, Cambridge, United Kingdom \\ ANDREW F. THOMPSON \\ California Institute of Technology, Pasadena, California
}

EMILY SHUCKBURGH

The British Antarctic Survey, Cambridge, United Kingdom

PETER H. HAYNES

The University of Cambridge, Cambridge, United Kingdom

(Manuscript received 30 August 2011, in final form 26 March 2012)

\begin{abstract}
Coherent jets are ubiquitous features of the ocean's circulation, and their characteristics, such as orientation and energetics, may be influenced by topography. In this study, the authors introduce a large-scale, topographic slope with an arbitrary orientation into quasigeostrophic, doubly periodic, barotropic and baroclinic systems. In both systems, the flow organizes itself into coherent tilted nonzonal jets that are aligned perpendicular to the barotropic potential vorticity (PV) gradient. In the two-layer system, the upper layer, the lower layer, and the barotropic PV gradients all have different orientations and therefore the jets cross the layer-wise PV gradients. The fact that the jets cross layer-wise PV gradients and the requirement of conservation of PV for fluid parcels together results in the drift of the tilted jets across the domain. Like their zonal counterparts, the tilted jets exhibit strong transport anisotropy. The dynamical response to jet deflection is very strong in the two-layer baroclinic case, with eddy energy production increasing by orders of magnitude as the topographic slope becomes more zonal. This increase in eddy energy is also reflected in an increase in jet spacing and a reduction in strength of the across-jet transport barriers, shown using an effective diffusivity diagnostic. The dynamics identified here, while formally valid within the constraints of quasigeostrophic scalings, provide important insight into the sensitive relationship between flow orientation and flow stability in regions with broad topographic slopes.
\end{abstract}

\section{Introduction}

A number of studies have identified and noted the potential impact of zonal jet structure (alternating eastward and westward velocities) on ocean circulation (Berloff et al. 2009; Maximenko et al. 2005), yet many instances of nonzonal jet orientation are also evident. Observations of sea surface height indicate jet cores at topographic gradient maxima (Hughes and Ash 2001) and also reveal increased eddy forcing of jets near

Corresponding author address: Emma Boland, DAMTP, Centre for Mathematical Sciences, Wilberforce Road, Cambridge CB3 OWA, United Kingdom.

E-mail: e.boland@damtp.cam.ac.uk topographically complex regions (Maximenko et al. 2005; Hughes 2005). Jets are known to be effective barriers to meridional transport near the surface from studies of the Southern Ocean (Marshall et al. 2006; Shuckburgh et al. 2009a,b). However, at middepths, diagnostics have shown enhanced mixing by eddies, (Abernathey et al. 2010), strongly linked to the interaction between topography and jets (Lu and Speer 2010).

One mechanism for the generation of nonzonal flow is topographic steering. This refers to the tendency of ocean currents to follow contours of $f / h$, where $f$ is the Coriolis parameter, and $h$ is depth, which are potential vorticity (PV) contours for a purely barotropic system (Marshall 1995). However, topographic steering may also impact more general barotropic and baroclinic 
stability properties of the flow. These processes are still not well understood in terms of their influence on transport and eddy-mean flow interactions. Several authors have investigated the dynamical effects of various types of bottom topography in numerical models, ranging from sinusoidal ridges (Thompson 2010) to an idealized Southern Ocean (Jackson et al. 2006), as well as simple quasigeostrophic models of the Antarctic Circumpolar Current (ACC) (see also Treguier and McWilliams 1990; Wolff et al. 1991; Treguier and Panetta 1994; Witter and Chelton 1998).

Understanding the processes involved in topography feedback on jet properties is of particular importance in the Southern Ocean: the flow of the ACC is known to be composed of several strong jets, as can be seen from observations of sea surface height through satellite altimetry (Sokolov and Rintoul 2007) and eddy-resolving numerical models [e.g. the Ocean Circulation and Climate Advanced Modeling (OCCAM) model, Lee and Coward (2003); the Modeling Eddies in the Southern Ocean (MESO) project, Hallberg and Gnanadesikan (2006)]. The ACC also passes through several topographically complex regions, such as the Drake Passage, the Macquarie Ridge, and the Campbell Plateau. These regions have been observed to introduce significant meridional perturbations to the flow (Gordon et al. 1978), resulting in many occurrences of nonzonal flows. Here we study how topographic steering and the propensity for the formation of coherent jets in the ocean combine to impact flow structure and transport properties. These dynamics are relevant to regions of the ocean such as the ACC, and thereby contribute to our overall understanding of ocean circulation, and its effect on the Earth's climate.

Historically, simplified models have provided insight into the dynamical processes that govern turbulent flows in the atmosphere and ocean. For example, previous investigations into both one- and two-layer models, such as Vallis and Maltrud (1993) and Panetta (1993), have shown the development of zonal jets as a result of the $\beta$ effect. The present study extends this previous work by considering a suite of doubly periodic barotropic and two-layer baroclinic turbulence simulations in which nonzonal jets form. Nonzonal jets are generated by the introduction of a bottom slope with both zonal and meridional components. Importantly, topographic steering in these simulations allows for a meridional component in the jets without the addition of any artificial forcing (cf. Smith 2007; Spall 2000; Arbic and Flierl 2004b). Our choice of a quasigeostrophic (QG) model is motivated by the key insight this system of equations has provided in similar flows. We acknowledge that in certain regions of the ocean, in particular the Southern
Ocean, topographic slopes may be larger than formal QG scalings. The impact of steeper slopes is addressed briefly in the conclusion, section 4 .

The introduction of a linear bottom slope into a baroclinically forced two-layer model was first investigated by Hart (1975) and Steinsaltz (1987), who analyzed the linear stability. LaCasce and Brink (2000) investigated the role of meridional slopes on wave and jet formation in a twolayer, $f$-plane model. They considered decaying turbulence whereas we focus on a forced-dissipative model. Thompson (2010) investigated the introduction of a meridional bottom slope in the presence of the $\beta$ effect as one of a range of bottom topographies for forced-dissipative turbulence-here we consider zonal components of a sloping bottom as well.

Our present model, in an effort to isolate key dynamics, is a significant simplification over real oceanographic situations. Yet sloped bottom topography has long been used in experimental fluid dynamics to introduce PV gradients, see, for example, Mason (1975), Whitehead et al. (1990), and Tamaki and Ukaji (2003), in which baroclinic effects in rotating tanks are investigated using applied density gradients. More recent experiments have also seen the formation of zonal jets in the presence of meridional slopes in such tanks (Bastin and Read 1998; Wordsworth et al. 2008), therefore aligning with the barotropic PV gradient, suggesting that the results of this study may apply to a broad range of flows.

The linear stability of quasigeostrophic models with zonally varying topography has been investigated in terms of free Rossby waves in, for example, Samelson (1992), Straub (1994), and Hallberg (1997), who find regimes of surface-bottom intensified modes in the presence of strong gradients, as well as barotropic regimes in the limit of weak topographic gradients. The strong topographic gradients required to create the trapped modes are not strictly valid in the quasigeostrophic approximation, however Hallberg (1997) reproduces some of the qualitative behavior in a primitive equation model. Tailleux and McWilliams (2000) also use a primitive equation model to investigate windforced Rossby waves over zonally varying topography, and find that waves over steep topography have enhanced phase speeds, and that the wind-forced response is enhanced.

We will now discuss our model configuration and compare it with other doubly periodic studies that have investigated nonzonal jets. Our barotropic simulations have a single PV gradient that rotates with the magnitude and orientation of the bottom slope. Our baroclinic, two-layer model, is more subtle, and the governing PV gradients are shown in Fig. 1a. The upper-layer background $\mathrm{PV}$ gradient $\mathbf{G}_{1}$ is fixed in the $y$ direction, defined 
a)

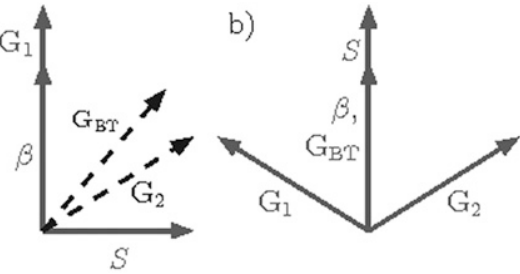

c)

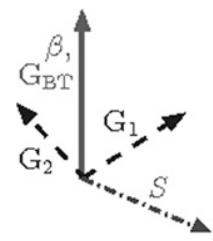

FIG. 1. Depiction in the $x-y$ plane of the configuration of the twolayer, doubly periodic, quasigeostrophic models used in (a) this paper, (b) Smith (2007), and (c) Arbic and Flierl (2004b). Thick lines denote that the quantity is fixed in direction, whereas dashed lines denote a quantity varied in direction, and the arrows point in the direction of the vector. Here, $\mathbf{S}$ denotes the overall shear of the background velocities driving the baroclinic instability, $\beta$ denotes planetary vorticity, $\mathbf{G}_{1,2}$ represent the individual layer potential vorticity gradients, and $\mathbf{G}_{\mathrm{BT}}$ the background barotropic potential vorticity gradient, as defined in (1) and (2), respectively, for (a). In (c), the dash-dot line for the shear indicates that while Arbic and Flierl could vary their shear direction, for most of the paper it was kept in one of two fixed directions. Note that in both (b) and (c), $\mathbf{G}_{\mathrm{BT}}$ and $\beta$ are fixed in the same direction.

by the planetary vorticity $\beta$ and the background velocity shear $\mathcal{U}$, which provides the source of potential energy from which baroclinic turbulence is generated. The lower-layer background PV gradient $\mathbf{G}_{2}$ varies with the magnitude and orientation of the slope in the bottom layer. This is not inconsistent with the doubly periodic nature of the domain as it is the depth gradient, not the absolute depth that alters the PV gradient. The doubly periodic domain can be thought of as representing a patch of the ocean far from boundaries, or a patch that is smaller than any externally enforced scale.

The background layer and background barotropic PV gradients are defined as follows:

$\mathbf{G}_{i}=\left(0, \beta+(-1)^{i-1} \frac{\mathcal{U}}{\lambda^{2}}\right)+\delta_{i 2}\left(h_{x}, h_{y}\right), \quad i=1,2, \quad$ and

$$
\mathbf{G}_{\mathrm{BT}}=\left(\frac{h_{x}}{2}, \beta+\frac{h_{y}}{2}\right),
$$

where $\delta_{i j}$ is the Kronecker delta function, subscript $i=1$ represents the upper layer, and $i=2$ the bottom layer, which are of equal depth. Potential energy is provided by a global shear vector, $\mathbf{S}=\mathbf{U}_{1}-\mathbf{U}_{2}$, and is then released through dynamic baroclinic instability. The domain-averaged layer-wise velocities $\mathbf{U}_{1}=(2 \mathcal{U}, 0)$ $\mathbf{U}_{2}=(0,0)$, provide basis states that are solutions to the quasigeostrophic equations in each layer. The evolution equations, the bottom gradients $h_{x}, h_{y}$, and the other parameters, are defined in section $2 \mathrm{~b}$. We find through our investigation that the introduction of a zonal $(x-)$ gradient in bottom slope leads to the production of tilted jets, steered as expected by PV conservation, but with the consequence that these jets flow across layer-wise PV gradients. While we keep the shear vector in our model purely zonal, others (Smith 2007; Arbic and Flierl 2004b) have investigated applying a nonzonal mean flow through a $\beta$ field in two-layer models, motivated by observation of such flows in the ocean.

Smith (2007) investigates forcing a doubly periodic two-layer quasigeostrophic model with a purely meridional mean flow. This configuration is illustrated in Fig. 1b for comparison with ours, where the fixed directions of the shear velocity, planetary vorticity, and layer-wise potential vorticities are shown in the $x-y$ plane. Smith's configuration produces what he describes as the "shear dispersion of potential vorticity;" the planetary PV acts to elongate zonally the eddies produced in the mean flow, leading to jet-like structure in cases with relatively strong $\beta$ and low bottom friction. He also notes an increase in barotropic kinetic energy with increasing $\beta$ while holding other variables fixed.

Arbic and Flierl (2004b) investigate a different parameter space again, once more in a doubly periodic two-layer quasigeostrophic model. By allowing arbitrary background velocities in either layer, they vary both the direction of the shear vector $\mathbf{S}$ and the angle between the layer PV gradients $\mathbf{G}_{1}$ and $\mathbf{G}_{2}$, as illustrated in Fig. 1c. Their results show clear zonal jets in setups with large values of $\beta$ and small angles between the layer PV gradients and like-signed vortices in those with smaller values of $\beta$ and larger angles between PV gradients. There are also notably some anisotropic structures, see especially their Figs. $6 c$ and $7 c$. Significantly, while the individual layer PV gradients can be varied (by varying the background shear velocities), shear components cancel such that the barotropic PV gradient is always only determined by $\beta$ and so is fixed in direction.

A significant difference between the configuration we present (shown in Fig. 1a) and those in Smith (2007) and Arbic and Flierl (2004b) is that, while their configurations require a prescribed nonzonal mean flow to try to imitate observed features, nonzonal jets arise in our configuration more naturally, we would argue, through the introduction of zonal bottom slopes in topography. Furthermore, a nonzonal mean flow is not a solution to the quasigeostrophic equations in Smith (2007), although Arbic and Flierl (2004b) ensure their shear vectors are solutions to their evolution equations. 
In addition, our configuration allows for variation in the directions of the barotropic and layer-wise PV gradients. We find that the anisotropic jets produced in the presence of a zonal slope in bottom topography align perpendicular to the direction of the barotropic PV gradient, rather than the individual layer gradients. Arbic and Flierl (2004b) find a maximum in eddy energy production when their overall shear is southward, or antialigned with the planetary PV gradient, which is similar to the configuration used by Smith (2007). While holding the shear fixed at two separate values and varying $\beta$ they similarly find an increase in eddy energy production with increasing $\beta$.

In section $2 \mathrm{a}$ we summarize the results of the onelayer model, and then in section $2 \mathrm{~b}$ set out the details of the two-layer model, and show some example results that demonstrate the formation of nonzonal jets. Section 3 contains analysis of the impact of the slope magnitude and orientation on the flow structure and statistical characteristics. We then compare and contrast the twolayer model results with the previous papers and comment on these in section 4 .

\section{Model}

\section{a. One-layer model}

We consider a one-layer fluid, taking quasigeostrophic approximations: small Rossby number, and small variations in the Coriolis parameter and depth. The system is described by the following equation:

$$
\frac{\partial Q}{\partial t}+J(\Psi, Q)=F_{0}^{2} \cos \left(k_{0} x\right)-\kappa \nabla^{2} \Psi+d
$$

Here, $J(\Psi, Q)$ is the Jacobian determinant of $\Psi$ and $Q$, the streamfunction and $\mathrm{PV}$ fields respectively, where the velocity $(u, v)=\left(-\Psi_{y}, \Psi_{y}\right)$. Here, $F_{0}^{2}$ is the amplitude of the forcing, which has a single zonal wavenumber $k_{0}, \kappa$ is the bottom friction, and $d$ is the small-scale dissipation included for numerical stability. The small-scale dissipation is implemented using a wavenumber filter, as described in the appendix of Smith et al. (2002). We force the system via sinusoidal forcing in the $y$ direction at a single $x$ wavenumber, first suggested by Kolmogorov (Arnold and Meshalkin 1960).

The PV $Q$ is defined in terms of the perturbation PV $q=\nabla^{2} \Psi$ and the background $\mathrm{PV}$ gradient $\mathbf{G}$ :

$$
\begin{aligned}
& Q=q+\mathbf{G} \cdot \mathbf{x}, \quad \text { and } \\
& \mathbf{G}=\left(h_{x}, \beta+h_{y}\right) .
\end{aligned}
$$

The background PV depends on the latitudinal variation of the Coriolis parameter $f=f_{0}+\beta y$ and the linear gradients of the bottom surface $h_{x}, h_{y}$, where

$$
h=\frac{f_{0}}{H}\left(\alpha_{1} x+\alpha_{2} y\right), \quad \alpha_{1,2}=\text { const. }
$$

where $H$ is the mean layer depth. From now on we will refer to $\beta^{*}=\beta+h_{y}$ for brevity. The resulting background PV structure can be seen in Fig. 2a. The effect of adding a topographic slope in the $x$-direction $h_{x}$ is equivalent to rotating the domain (and modifying the magnitude of $\beta$ ). Still, this is not a trivial transformation since the forcing has a highly anisotropic horizontal structure and the alignment of this structure relative to the barotropic PV gradient changes. The system is nondimensionalized using $F_{0}$ and $k_{0}$ as follows: $\Psi^{\prime}=k_{0}^{2} \Psi / F_{0}, q^{\prime}=q / F_{0}, \kappa^{\prime}=\kappa / F_{0}, \beta^{\prime}=\beta / k_{0} F_{0}, h^{\prime}=$ $h / F_{0}$. From now on we will drop the 's. For all the simulations presented, the domain is a square of length $32 \pi / k_{0}$ and calculations are made on a square grid of size $256 \times 256$. We hold the bottom friction fixed throughout, $\kappa=0.01$.

Example fields from the one-layer model can be seen in Fig. 3, which shows snapshots of the streamfunction $\Psi$ and the perturbed $\operatorname{PV} q$, respectively, at a time after statistical equilibrium has been reached, for three different values of $h_{x}$. In these runs, $\beta^{*}=0.5$. Coherent jets are observed in all three snapshots, although the jets are not purely zonal in nature when $h_{x}$ is nonzero. As the magnitude of the zonal component of the slope increases, the background PV gradient rotates. The jets rotate, or tilt, as well, to align perpendicular to the PV gradient, which is the only one in the system. The forcing still imposes directionality on the flow since it has a fixed orientation, see (3). The jet tilt is largely a response to the changing orientation of the background PV gradient G. Dynamically the jet formation process is no different to the flat-bottom case. We present these results primarily to contrast them with the response in the two-layer, baroclinic simulations.

Figure 4a shows the observed angle of jet tilt from the numerical simulation $\phi_{\text {jet }}$, calculated by taking the ratio of the meridional to zonal wavenumbers corresponding to the maximum amplitude of the power spectrum of the PV perturbation $q$, time averaged after statistical equilibrium had been reached, against the theoretical angle $\phi_{\mathrm{BT}}$, defined as perpendicular to the PV gradient:

$$
\phi_{\mathrm{BT}}=\tan ^{-1}\left(\frac{h_{x}}{\beta^{*}}\right)
$$



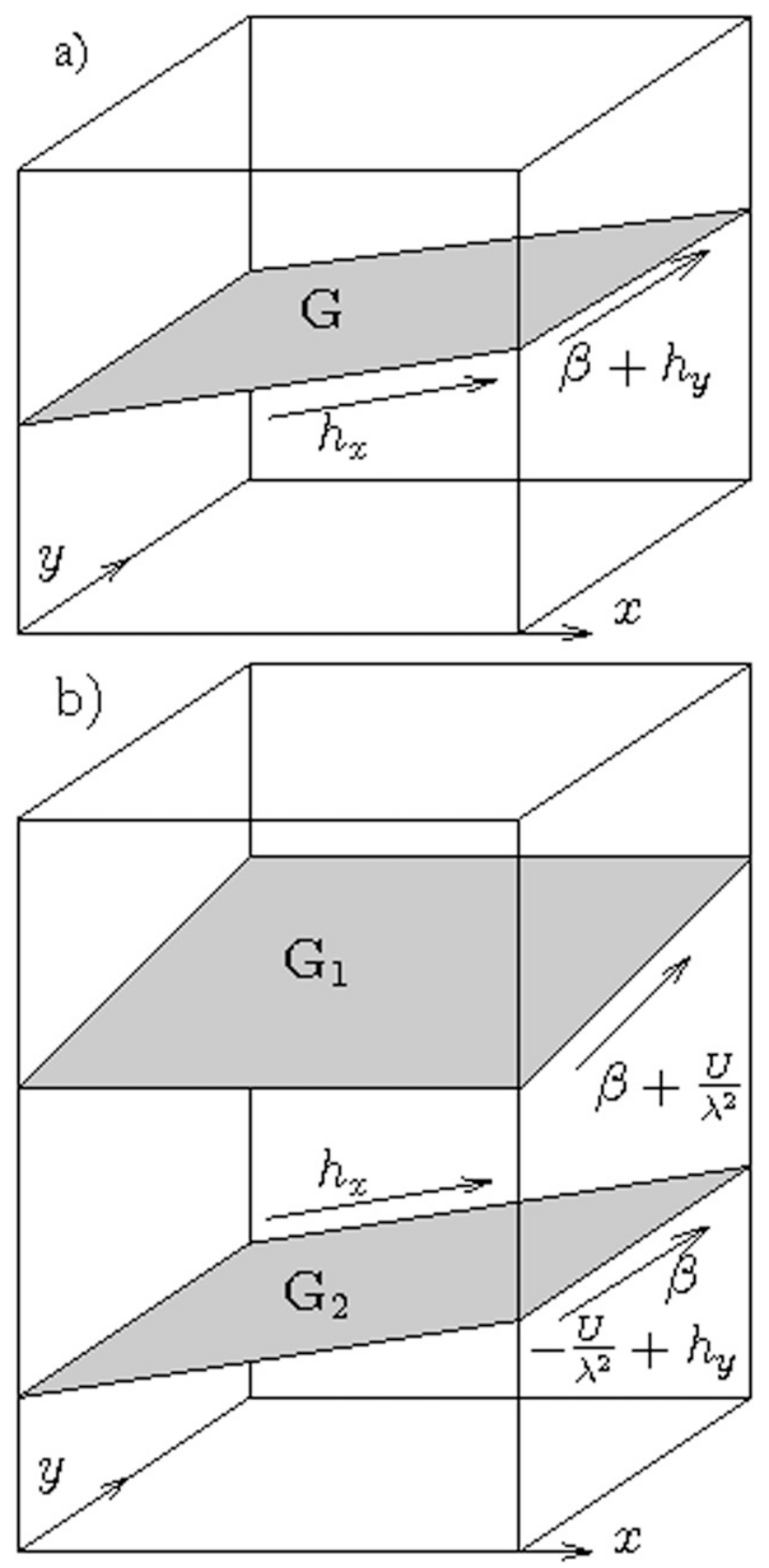

FIG. 2. Depiction of the background (as opposed to perturbation) PV structure of the (a) one- and (b) two-layer models. The labels refer to the quantities defined in Eq. (5) for (a) and Eq. (1) for (b).

Both angles are defined such that $\phi=0$ is equivalent to purely zonal flow. The excellent fit confirms that the jets align perpendicular to the PV gradient to conserve PV. Note that the introduction of the bottom slopes does not act to rotate the entire problem, as the sinusoidal forcing still varies only in the zonal direction; however, this effect is apparently small compared to the system's organization into coherent jets.

\section{b. Two-layer model}

While the two-layer model represents a moderate increase in complexity, the potential for baroclinic instability to be active produces a dramatic difference from the barotropic simulations. No explicit small-scale forcing is required, and following the work of Haidvogel and Held (1980), Panetta (1993), and Held and Larichev (1996), we constrain the system to a global domainaveraged shear between the two layers $\mathbf{S}$, as previously defined. We define two streamfunctions for each layer, $\Psi_{i}$ and $\psi_{i}$, related by $\Psi_{i}=\psi_{i}+\mathbf{U}_{i} \cdot(-y, x)$, where $\mathbf{U}_{i}$ are defined in section 1 such that there is a permanent, spatially uniform zonal shear between the two layers throughout the domain. Note that this velocity structure is a solution of the equations:

$$
\frac{\partial Q_{i}}{\partial t}+J\left(\Psi_{i}, Q_{i}\right)=-\delta_{i 2} \kappa \nabla^{2} \Psi_{i}+d, \quad i=1,2,
$$

where all symbols are as before. The potential vorticities are given by

$$
\begin{aligned}
Q_{i} & =q_{i}+\mathbf{G}_{i} \cdot \mathbf{x}, \quad i=1,2, \quad \text { and } \\
q_{i} & =\nabla^{2} \psi_{i}+\frac{1}{2 \lambda^{2}}\left[\psi_{(3-i)}-\psi_{i}\right], \quad i=1,2,
\end{aligned}
$$

where the deformation radius, $\lambda=\sqrt{g^{*} H} / f_{0}, g^{*}=g\left(\rho_{2}-\right.$ $\left.\rho_{1}\right) / \rho_{0}$ is the reduced gravity at the internal interface in terms of the layer densities $\rho_{1,2}$ and a reference density $\rho_{0}$. Here, $\mathbf{G}_{i}$ is defined in Eq. (1), $H$ is the overall depth of the system, and the layers are of equal depth $H / 2$. The resulting background PV structure can be seen in Fig. $2 \mathrm{~b}$. The equations are nondimensionalized using $\lambda$ and $\mathcal{U}: \psi_{i}^{\prime}=\psi_{i} / \mathcal{U} \lambda, q_{i}^{\prime}=\lambda q_{i} / \mathcal{U}, \kappa^{\prime}=\lambda \kappa / \mathcal{U}, \beta^{\prime}=\lambda^{2} \beta / \mathcal{U}$, resulting in the following system of equations, where we have dropped the 's and $d$ :

$$
\begin{gathered}
q_{1 t}+(\beta+1) \psi_{1 x}+2 q_{1 x}+J\left(\psi_{1}, q_{1}\right)=0, \quad \text { and } \\
q_{2 t}+\left(\beta-1+h_{y}\right) \psi_{2 x}-h_{x} \psi_{2 y}+J\left(\psi_{2}, q_{2}\right)=-\kappa \nabla^{2} \psi_{2} .
\end{gathered}
$$

The perturbations $\psi_{i}$ about the background flow are stepped in time using a third-order Adams-Bashforth scheme. For all the simulations presented, the domain is a square of length $128 \lambda$ and calculations are made on a square grid of size $256 \times 256$ (higher-resolution simulations were tested and showed similar results). We hold the bottom friction fixed throughout, $\kappa=0.1$, chosen to be small enough to allow the generation of clear jets but large enough to statistically equilibrate the system—see, for example, Arbic and Flierl (2004a). 

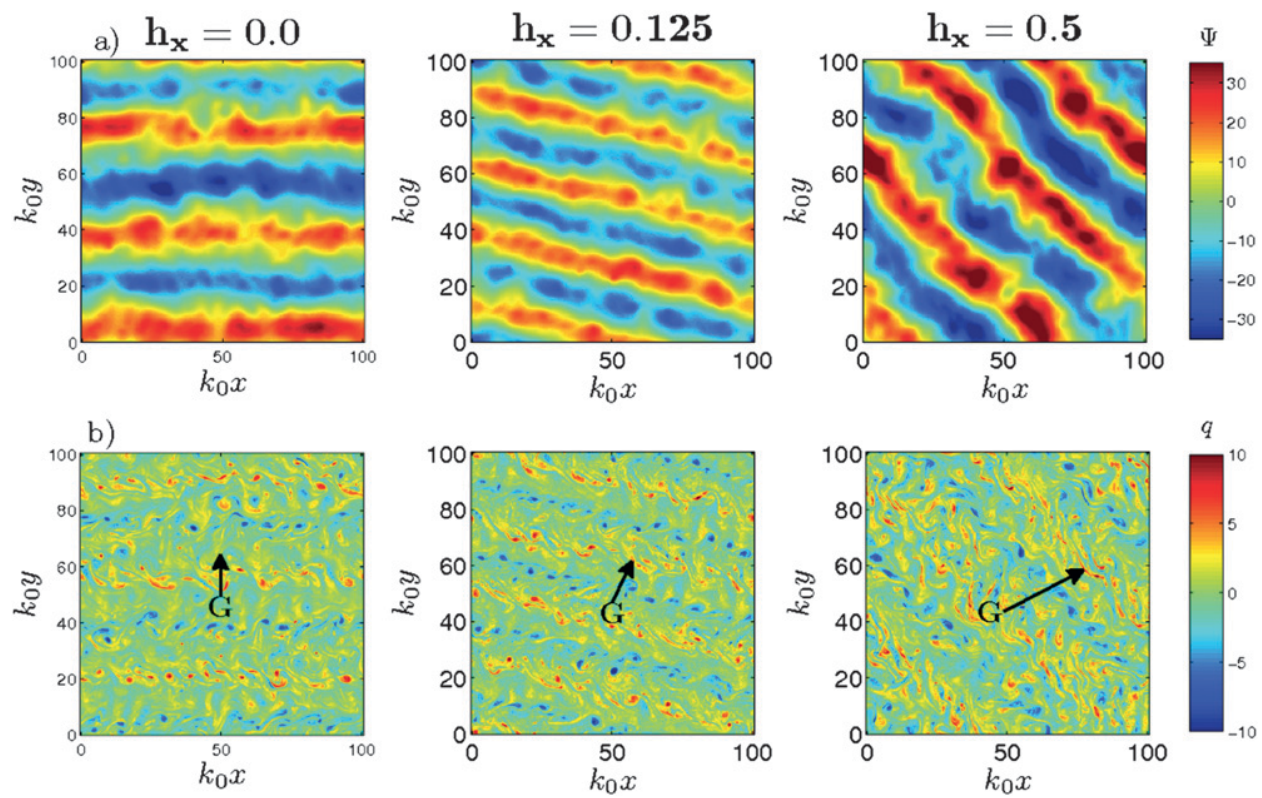

FIG. 3. Snapshots of one-layer (a) streamfunctions $(\Psi)$ and (b) PV perturbations $(q)$ during statistical equilibrium, for three values of $h_{x}: 0.0,0.125$, and 0.5 . In these simulations $\beta^{*}=0.5$. The tilting of the jets is associated with the changes in the PV structure caused by the introduction of a zonal gradient in bottom slope, $h_{x}$. (bottom) The direction of the background PV gradient $\mathbf{G}$ is depicted for each run by the black arrow.

Example fields from the two-layer model can be seen in Fig. 5, which shows snapshots of the perturbed upper and lower layer PV, $q_{1}$ and $q_{2}$ respectively, at a time after statistical equilibrium has been reached, for three values of $h_{x}$, alongside histograms of the total PV's, $Q_{1}$ and $Q_{2}$, respectively. In this run the parameters are set at $\beta=0.75$ and $h_{y}=0.0$. Again, nonzonal jet structure is observed. Despite the differing background PV structure in both layers [see (9) and Fig. 2], the upper- and lower-layer perturbed PV fields are identical in the orientation of their jets.

Probability density functions (PDFs) of PV have been used in previous work, such as Marshall et al. (1993) and Thompson et al. (2010), to identify regions of high and low mixing. Intense mixing leads to PV homogenization, resulting in a high probability at the PV associated with that region, and conversely transport barriers are associated with sharp gradients in $\mathrm{PV}$, and so low probability at the relevant PV value. Throughout this paper we use time-mean histograms (unnormalized PDFs) of total PV to gain insight into the transport properties of the numerical simulations. As discussed in section 3a, in some simulations with tilted jets, the doubly periodic domain means that there is only one unique jet in the domain. In these cases, we take advantage of this feature by remapping all $\mathrm{PV}$ values into the unique range of $\mathrm{PV}$ covered by one jet before producing the histogram.
When plotting these, we repeat the histogram to reproduce the number of jets and the full range of $\mathrm{PV}$ in one domain, for purposes of comparison.

In the case where $h_{x}=0.0$ (the left-hand plots in Fig. 5), classic zonal jets can clearly be seen in both layers, though they are stronger in the upper layer, which has a stronger background PV gradient. The PV histogram shows minima in both layers associated with the sharp PV gradients present at the core of the jets. In the top layer, weaker transport barriers associated with the westward jets are also present- these can be seen in the total PV histogram and are correlated with the features that can just be seen between the jet structures in the snapshot. Westward jets are a robust feature in two layer quasigeostrophic simulations that are also observed to be transport barriers by, for example, Beron-Vera et al. (2008).

While the relationship between the direction of the flow and the PV gradients will be investigated in section 3 , it is immediately apparent that, in the top layer at least, the jets are crossing the local PV gradient in cases where $h_{x}$ is nonzero, as the top layer PV gradient is always in the meridional direction (see Fig. 1). The total $\mathrm{PV}$ histograms confirm that for these cases, the PV is rather homogeneous, that is, with no strong spatial structure, in both layers, without the sharp minima seen in the $h_{x}=0.0$ case, implying more strongly homogenized PV. 
a)

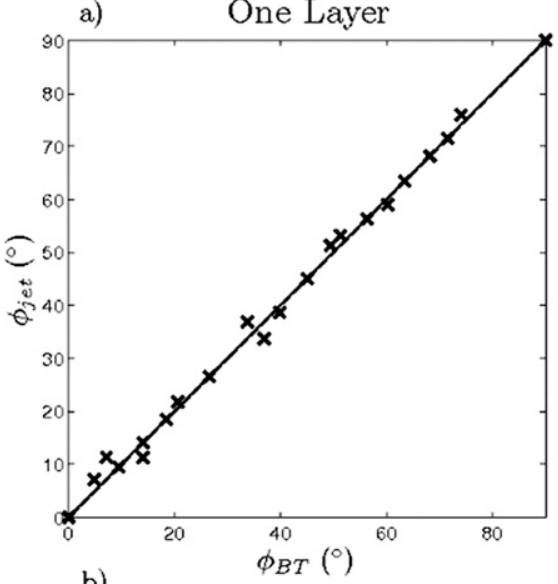

b)

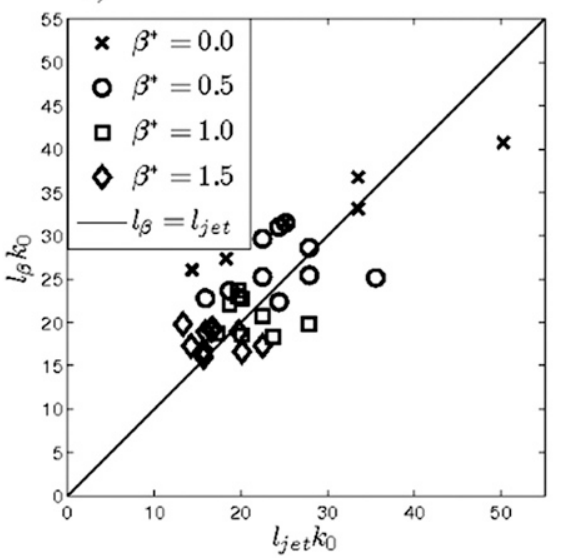

Two Layer
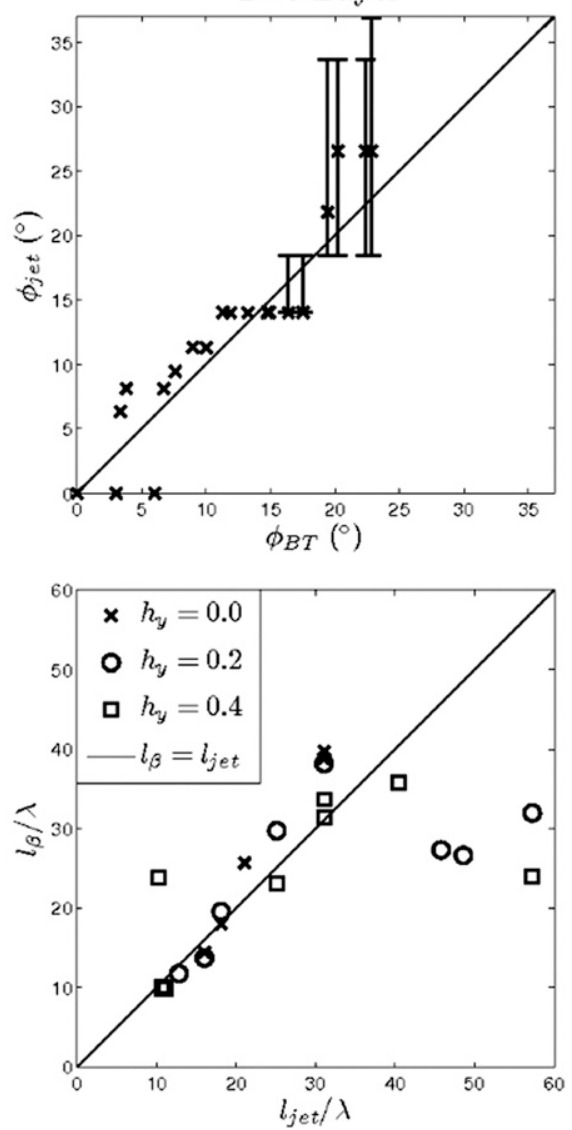

FIG. 4. (a) A comparison between observed jet tilt $\phi_{\text {jet }}$ and the predicted tilted $\phi_{\mathrm{BT}}$ for oneand two-layer cases. Here, $\phi_{\text {jet }}$ is found from the power spectrum of the PV field $q$ and the upper-layer PV field $q_{1}$ for the one- and two-layer simulations, respectively; $\phi_{\mathrm{BT}}$ is defined in (7) and (13). Symbols indicate the modes of the measured angle $\phi_{\text {jet }}$, and the error bars indicate maxima and minima, after statistical equilibrium - the angles in the one-layer simulations, and many two-layer simulations, remained constant in time so have no error bars. (b) A comparison between observed jet spacing $l_{\text {jet }}$ and the Rhines scale $l_{\beta} . l_{\text {jet }}=L \cos \left(\phi_{\text {jet }}\right) / n_{\text {jet }}$, where $L$ is the length of the domain, and $n_{\text {jet }}$ is the number of jets crossing the $y$ axis; $l_{\beta}$ is defined in Eq. (14). The different symbols indicate simulations with different values of $\beta *$ or $h_{y}$.

\section{Results}

\section{a. Jet deflection}

When analyzing the angle of jet deflection, it is important to recognize that the doubly periodic nature of the domain results in some restrictions on the possible deflection angles $\phi_{\text {jet }}$ for a given number of jets, in both the one- and two-layer cases. For a given number of jets, there are set angles at which re-entry at the beginning of the domain is possible. In fact, for $n$ jets crossing the $y$ axis in one domain, the tangent of the angle must be equal to $m / n$, where $m$ is the number of jets crossing the $x$ axis, see Fig. 6 . While this applies to both models, this can be seen most clearly in the stepped nature of the two-layer results in Fig. 4a, partly because the two-layer runs cover a smaller range of angles, and so the effect appears larger. We do not believe that this angle quantization affects our results, given that Fig. 4a clearly shows that our simulations cover a range of alignments with their preferred direction.

Taking $m$ and $n$ to be in the range $0-16$, as is found for the two-layer runs investigated, the allowed values of $\phi$ and the separation between the jets $l_{\text {jet }}=L / \sqrt{n^{2}+m^{2}}$, where $L$ is the length of the domain, can be seen in Fig. 7, with the actual points for the two-layer results highlighted. See Fig. 6 for the geometry of these quantities. A purely zonal jet would have $m=0$ and so $\phi=0^{\circ}$. A purely meridional jet would have $n=0$ and so $\phi=90^{\circ}$. Note that for a given value of $m$ and $n$, there are $n$ unique jets only if $m$ is a multiple of $n$ or vice versa ( $m$ unique 
a)
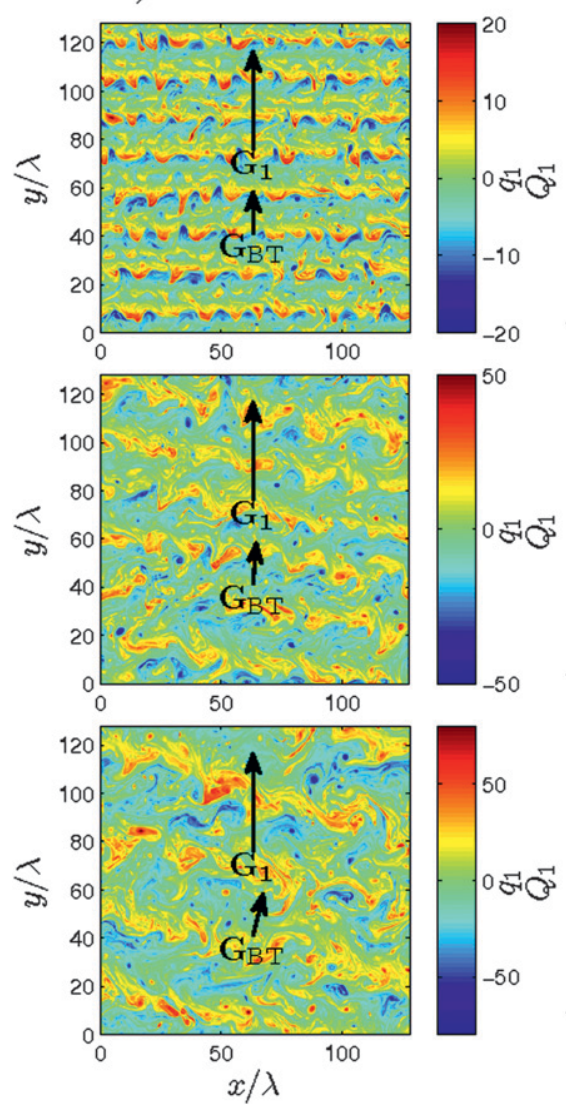
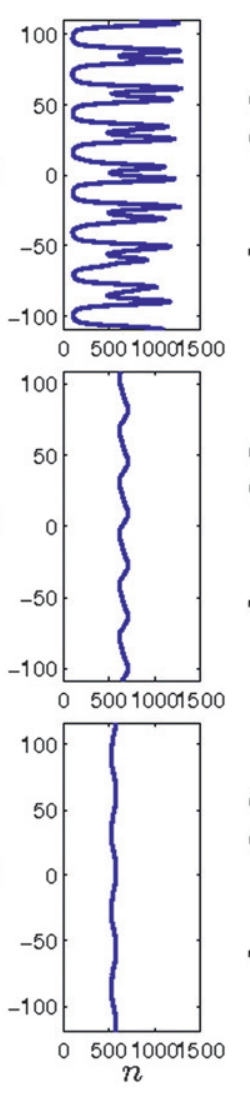

b)

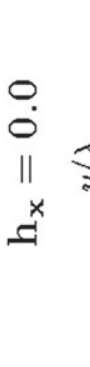

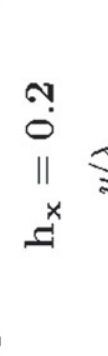

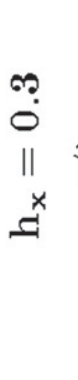

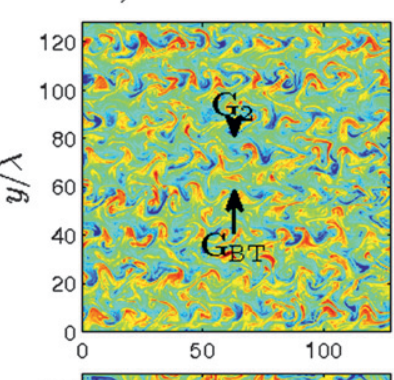
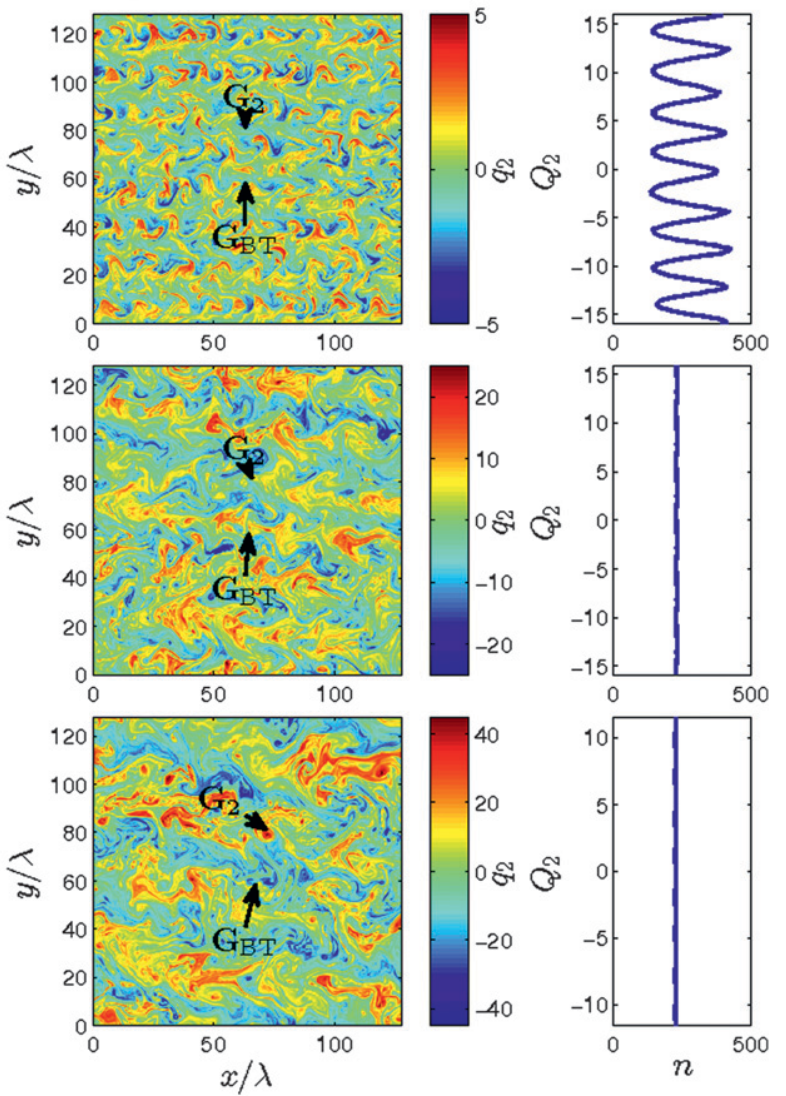

FIG. 5. Snapshots of two-layer (a) upper- and (b) lower-layer PV perturbations $q_{1}$ and $q_{2}$ during statistical equilibrium, for various values of $h_{x}$, alongside time-mean histograms of the total PV, $Q_{1}$, and $Q_{2}$, respectively, see Eq. (9). In these simulations, $h_{y}=0.0$ and $\beta=0.75$. The tilting of the jets is associated with the changes in the lower-layer PV structure, $q_{2}$, caused by the introduction of a zonal gradient in bottom slope $h_{x}$. The sharp minima in the total PV histograms for the $h_{x}=0.0$ case is associated with steep PV gradients at the core of the jets. The relative homogenization shown in the other histograms shows that the jets cross layer-wise PV gradients in these cases. In the cases where $h_{x} \neq 0$, we take advantage of the PV mapping technique described in section 3a to produce the histograms. The magnitude and direction of the layer-wise background PV gradients $\mathbf{G}_{1}$ and $\mathbf{G}_{2}$ are shown along with the background barotropic PV gradient $\mathbf{G}_{\mathrm{BT}}$ by black arrows.

jets if $n$ is a multiple of $m$ ). Otherwise, there is only one unique jet, as the doubly periodic nature of the domain means that what might appear to be distinct jets are in fact all parts of a single jet that wraps around the domain.

In the two-layer case, the jets also deflect from purely zonal when a zonal bottom slope $h_{x}$ is introduced. Motivated by the one-layer model, which can be interpreted as the barotropic mode of a multilayered model in the limit of relatively weak slopes, we look for a relationship between the gradient of the mean barotropic potential vorticity $\mathbf{G}_{\mathrm{BT}}$, defined in (2), and the angle of deflection $\phi_{\text {jet }}$, calculated as previously from the power spectrum of the upper-layer PV perturbation field $q_{1}$. Calculations using the equivalent lower-layer field $q_{2}$ produce identical results. Once again, a strong linear fit is found, which can be seen in Fig. 4, where the theoretical angle,

$$
\phi_{\mathrm{BT}}=\tan ^{-1}\left(\frac{h_{x} / 2}{\beta+h_{y} / 2}\right) \text {, }
$$

is perpendicular to $\mathbf{G}_{\mathrm{BT}}$.

Thus, in both cases it can be seen that the jets tend to align perpendicular to the barotropic PV gradient. In the one-layer case, this is the only PV gradient there is, and the system appears to better conform to the angle, shown by the tight fit in Fig. $4 \mathrm{a}$ and the change in jet spacing seen in Fig. 3. The fit is not as strong in the twolayer case, (see Fig. 4), and the system shows an increase in the jet spacing as $h_{x}$ increases, (see Fig. 5), which is discussed in section $3 b$.

The hypothesis that the jets align perpendicular to the barotropic PV gradient $\mathbf{G}_{\mathrm{BT}}$ can be further confirmed by 


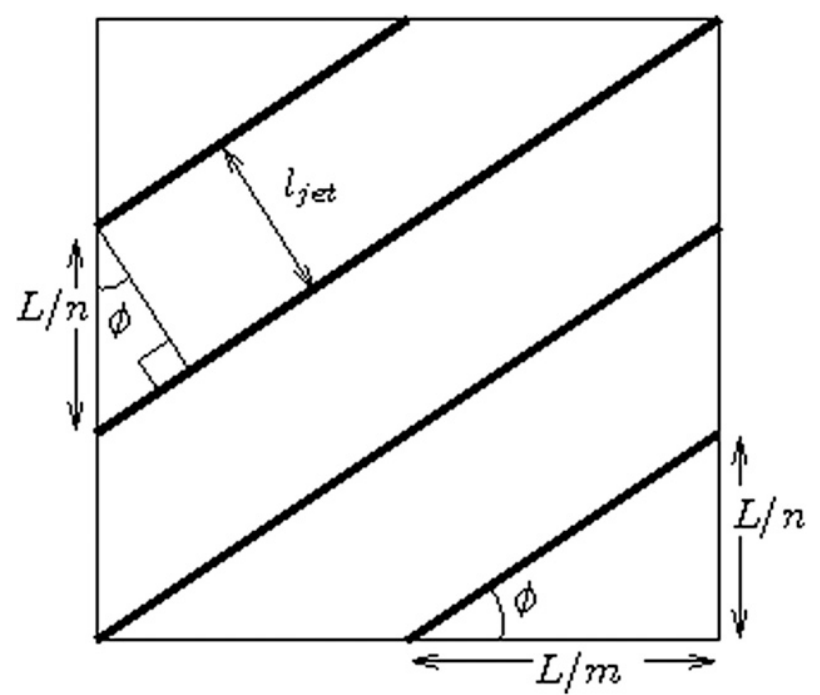

FIG. 6. Example geometry for a simulation in a domain of size $L$ by $L$ with $n=3$ jets crossing the $y$ axis and $m=2$ jets crossing the $x$ axis. We can see that $\cos \phi=l_{\text {jet }} n / L=n / \sqrt{n^{2}+m^{2}}$, and therefore that the jet spacing, $l_{\mathrm{jet}}=L / \sqrt{n^{2}+m^{2}}$.

looking at the total barotropic PV fields for the twolayer simulations. Figure 8a shows snapshots of total barotropic PV for each of three simulations with $h_{x}=$ $0.0,0.2$, and 0.3 , respectively, alongside mean histograms of the total barotropic PV. In the two simulations with $h_{x}=0.2$ and 0.3 , as both simulations have 1 jet crossing the $x$ axis, that is, $m=1$, there is only one unique jet in each simulation that wraps around the domain multiple times because of the double periodicity, as discussed previously. We can take advantage of this fact to improve the resolution of the histograms by remapping all the values of the total barotropic $\mathrm{PV}$ into a range of the unique PV values for these two simulations. This results in a histogram of a single jet, which for comparison has been replotted $n$ times. It can be seen, in comparison with Fig. 5, that there are clear eddy transport barriers associated with the jets in all three cases, characterized by minima in the PV histograms. Thus, the barotropic PV gradient is providing the dominant direction for the system, and the barotropic PV structure is composed of coherent jets, which show clear signs of anisotropy.

\section{b. Jet spacing}

Panetta (1993) and Thompson (2010) showed that in two-layer models such as these, the jet spacing is given by a Rhines scale dependent on the eddy velocity $V$ and the magnitude of the planetary vorticity gradient $\beta$. In this case, we consider the relevant gradient to be $\mathbf{G}_{\mathrm{BT}}$, the background barotropic PV gradient, equal to $\mathbf{G}$ in the one-layer case, as defined in (5), and as defined in
(2) in the two-layer case, and equal to $\beta$ in both Panetta (1993) and Thompson (2010):

$$
l_{\beta}=2 \pi \sqrt{\frac{V}{\left|\mathbf{G}_{\mathrm{BT}}\right|}},
$$

where $l_{\beta}$ is the Rhines scale. In the two-layer case, we use the eddy velocity $V=\sqrt{\left\langle\left|\nabla \psi_{1}^{\prime}\right|^{2}+\left|\nabla \psi_{2}^{\prime}\right|^{2}\right\rangle}$, and this seems to fit the data well, as can be seen in the right-hand plot in Fig. 4b. The fit is not as close at larger jet separations, although we believe this is related to the jet quantization effect discussed in section 3a-compare to Fig. 7. This supports the identification of the barotropic PV gradient $\mathbf{G}_{\mathrm{BT}}$ as dominating in determining the geometric properties of the jets in the simulations. For the one-layer case, the eddy velocity does not give a good agreement with the Rhines scale, but we find that the rms of the along jet velocity $\left\langle u_{\text {jet }}^{2}\right\rangle^{1 / 2}$ fits well, see the left-hand plot in Fig. 4b. ${ }^{1}$ An overbar indicates, here and throughout, an along-jet mean, and primes indicate that the along-jet mean has been removed, that is, $\psi^{\prime}=\psi-\bar{\psi}$. The angular brackets indicate an integration over turbulent fluctuations and the doubly periodic domain.

The results of section 3a show that an increase in $h_{x}$ leads to an increase in angle and an increase in the jet spacing for the two-layer case. Taking into account that increasing $h_{x}$ acts to strengthen $\left|\mathbf{G}_{\mathrm{BT}}\right|$, and that the twolayer system has been seen to obey the Rhines scaling as defined in (14), it can be deduced that when $h_{x}$ increases there must be a larger increase in the velocity scale, associated with the eddy velocity. This is investigated in the next section, which looks at how the eddy energy production of the system varies with our parameters.

\section{c. Eddy energy production}

By manipulation of (8), we can find the following expression for the energy balance in the two-layer case:

$$
\begin{aligned}
& \left\langle\frac{1}{2} \frac{\partial}{\partial t}\left[\left|\nabla \psi_{1}\right|^{2}+\left|\nabla \psi_{2}\right|^{2}+\left(\psi_{1}-\psi_{2}\right)^{2}\right]\right\rangle+\left\langle\psi_{1} \psi_{2 x}\right\rangle \\
& \quad=-\kappa\left\langle\left|\nabla \psi_{2}\right|^{2}\right\rangle+d
\end{aligned}
$$

The first (time varying) term represents the total energy of the system, split into kinetic energy terms (the first two terms) and a potential energy term. Neglecting $d$ as

\footnotetext{
${ }^{1}$ In the one-layer case we believe that the sinusoidal forcing is affecting the length scale for the eddies, leading to little variation in eddy velocity between different setups.
} 


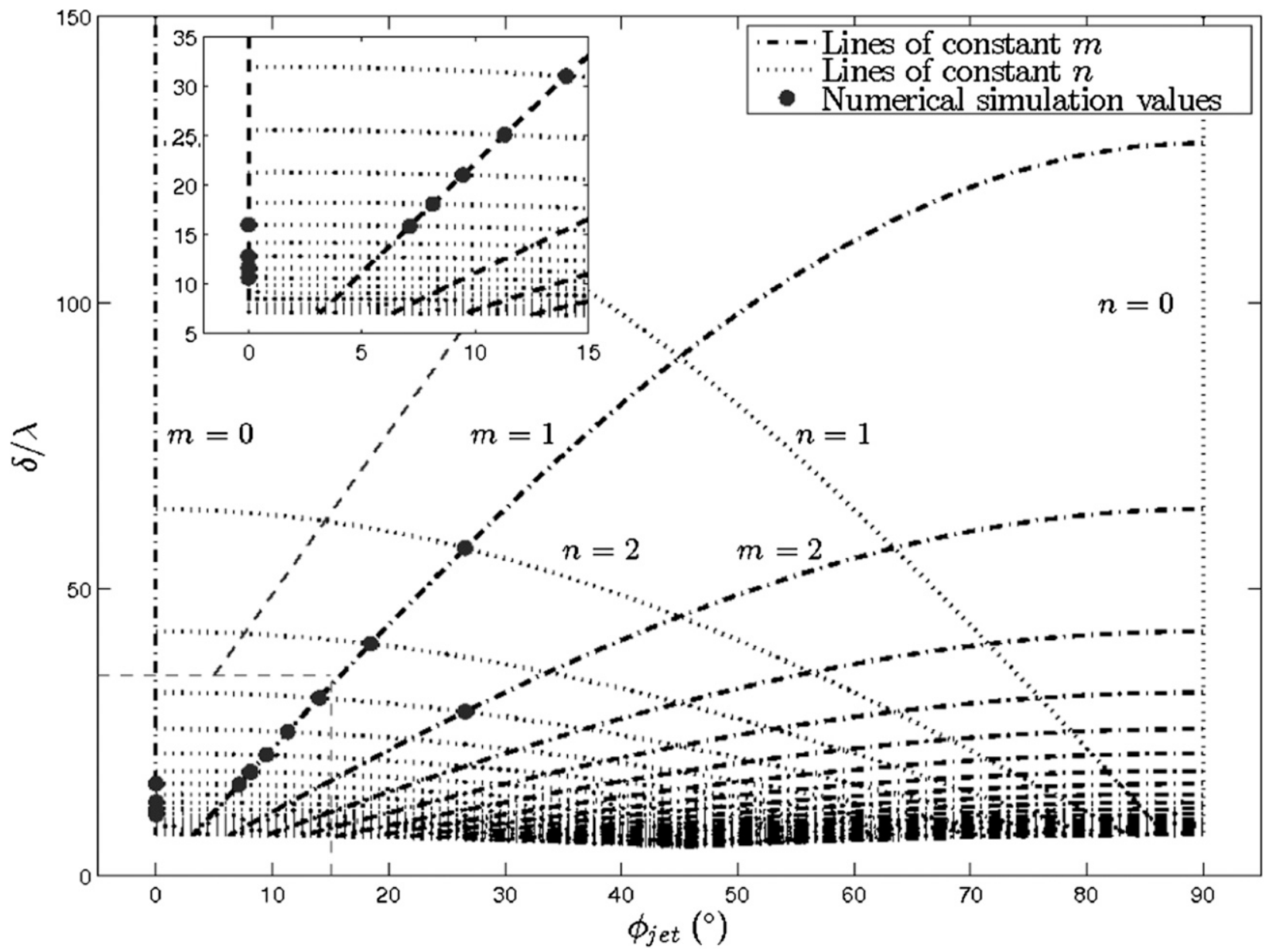

FIG. 7. Allowed jet separations $\delta / \lambda$ vs jet angle $\phi_{\text {jet }}$, where $\tan \phi_{\text {jet }}=m / n$, where $m$ and $n$ are in the range $0-18$, for a doubly periodic domain. Dotted lines indicate constant $n$, and dash-dot lines constant $m$; these lines cross at integer pairs of $m$ and $n$. The dots highlight the values for our two-layer numerical simulations. The finite size of the domain provides fewer and fewer options at large separations and large angles. This shows that the system appears to increase $\delta\left(l_{\text {jet }}\right)$ with increasing $h_{x}$, which corresponds to increasing $\phi_{\text {jet }}$, associated with increased eddy energy production, see section $3 \mathrm{c}$.

it makes a relatively small contribution, then at statistical equilibrium the eddy energy production $\varepsilon \equiv \psi_{1} \psi_{2 x}$ is balanced by the loss to bottom friction. The variation of $\varepsilon$ with $h_{x}, h_{y}$, and $\beta$ can be investigated by taking the time mean and standard deviation of $\varepsilon$ once statistical equilibrium has been reached, by measuring $\kappa\left\langle\left|\nabla \psi_{2}\right|^{2}\right\rangle$. Note that none of these three parameters enter into the energy balance directly (see Thompson and Young 2007), and the nondimensionalization by $\mathcal{U}$ removes any dependence on forcing. It should be noted that while Smith (2007) calculates the kinetic energy, and Arbic and Flierl (2004b) calculate the total energy of the system, these are both intrinsically related to our $\varepsilon$ after statistical equilibrium has been reached, as seen from (15), and the qualitative behavior of the results found do not change if we use kinetic energy or total energy instead.
To compare with the work of Arbic and Flierl (2004b), as discussed in section 1, we calculate the angle between $\mathbf{G}_{\mathrm{BT}}$ and $\mathbf{S}$, defined as

$$
\theta_{\mathrm{BT}}=\tan ^{-1}\left(\frac{\beta+h_{y} / 2}{h_{x} / 2}\right)
$$

Thus, $\theta_{\mathrm{BT}}=90^{\circ}$ corresponds to $\mathbf{G}_{\mathrm{BT}}$ being perpendicular to the fixed zonal shear $\mathbf{S}$, resulting in zonal jets, and $\theta=$ $0^{\circ}$ would correspond to the two vectors being parallel, (N.B. $\theta_{\mathrm{BT}}=90^{\circ}-\phi_{\mathrm{BT}}$ ). For each numerical simulation, once statistical equilibrium is reached, the system is allowed to progress for at least twice the time taken to reach statistical equilibrium, then the time mean and standard deviation of $\varepsilon$ is taken. Figures $9 \mathrm{a}$ and $9 \mathrm{~b}$ show an increase in eddy energy production with increasing $h_{x}$ 

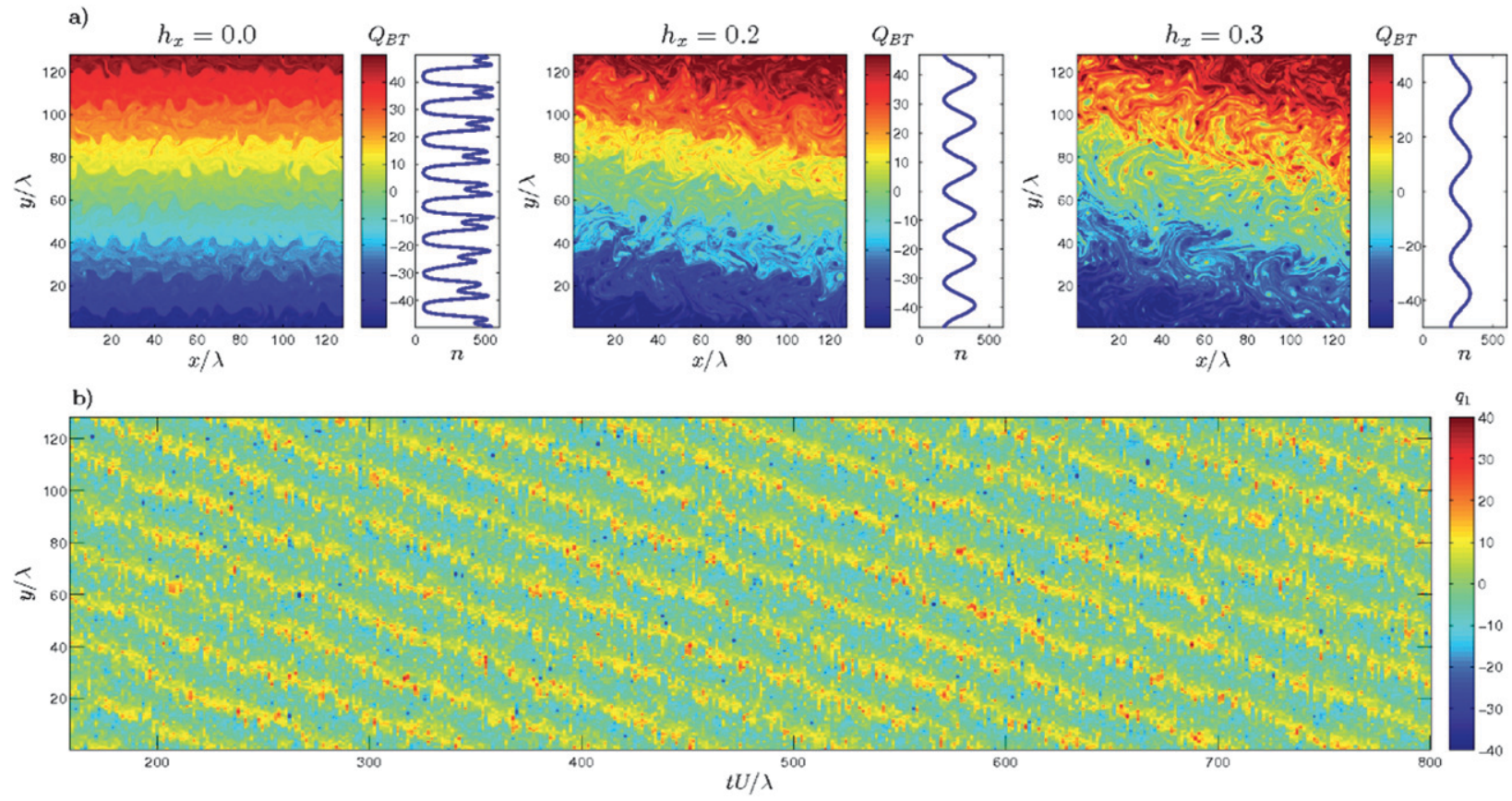

FIG. 8. (a) Snapshots and mean histograms of total barotropic potential vorticity $Q_{\mathrm{BT}}$ for two-layer runs with $h_{x}=0.0,0.2$, and 0.3 . Minima in the histograms correspond to the sharp gradients in PV at the center of jets. (b) Hovmöller diagram showing the nondimensional upper-layer perturbation PV field, $q_{1} \lambda / \mathcal{U}$, at $x / \lambda=0$ vs time $(t \mathcal{U} / \lambda)$ for a two-layer simulation with $\beta=0.75, h_{y}=0$ and $h_{x}=$ 0.1. The jets, characterized by the maxima in the PV gradient, can be seen to be moving with a constant speed in the negative $y$-direction.

for all values of $h_{y}$, with a particular jump in the $h_{y}=0.4$ series between $h_{x}=0.2$ and 0.3 , but no clear pattern with increasing $h_{y}$. A clear relationship of increasing energy as $\theta_{\mathrm{BT}}$ decreases is seen in Fig. 9c, although it does not hold at the lower values plotted.

Thus, these results confirm the previous finding, in section $3 b$, that the system shows a large increase in eddy velocity with increasing $h_{x}$, which results in an increase in the Rhines scale and so the jet spacing, even while the total magnitude of the barotropic PV gradient, $\mathbf{G}_{\mathrm{BT}}$, is increasing. The two-layer system shows a change in energies over several orders of magnitude, which we postulate is due to the across-PV gradient jets and the subsequent increase in relative vorticity in order to conserve PV. This is discussed further in section 3e.

The effect of increasing $h_{x}$ is to move the direction of the barotropic PV gradient $\left(\mathbf{G}_{\mathrm{BT}}\right)$ closer to the direction of the background shear (see Fig. 1). Thus the increase of eddy energy production with $h_{x}$ and the overall trend in the relationship with $\theta_{\mathrm{BT}}$ is consistent with Arbic and Flierl (2004b), who found a maximum in energy when $\theta_{\mathrm{BT}}=180^{\circ}$ (assuming that the pattern seen in Fig. 9c would be symmetric about $\theta_{\mathrm{BT}}=90^{\circ}$, as has been found in a few test cases). It appears that at high values of $\theta_{\mathrm{BT}}$ there is a large decrease in the magnitude of $\varepsilon$ as $h_{y}$ is increased, even though $\theta_{\mathrm{BT}}$ changes very little. This is due to the stabilizing effect of $h_{y}$, similar to that of $\beta$ (Thompson 2010).

Several simulations at higher resolution were carried out to assess any resolution dependence; however the results found were indistinguishable from the equivalent lower resolution simulations.

\section{d. Jet drift}

During analysis of the results, it was noted that simulations with tilted jets exhibited "jet drift," that is, the jets present changed their position within the domain over time. An example Hovmöller (space-time) diagram for a two-layer simulation with $\beta=0.75, h_{y}=0$, and $h_{x}=$ 0.1 can be seen in Fig. 8 b.

We postulate that this is due to the alignment of the jets in a direction perpendicular to the barotropic PV gradient, resulting in jets that are not perpendicular to the PV gradients in individual layers. Therefore, there is systematic advection of PV and, potentially, the systematic growth of PV anomalies aligned with the jets. The system compensates through continuous displacement of the jets, which in particular means that the longtime average velocity at any location in the direction parallel to the PV gradient in a layer is zero. In the upper layer the PV gradients are in the $y$ direction, therefore the angle $\phi_{\text {jet }}$ between the jet direction and the $x$ axis is 
a)
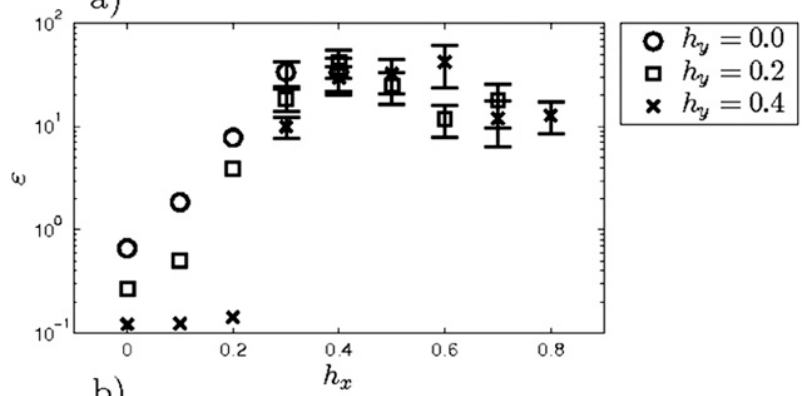

b)

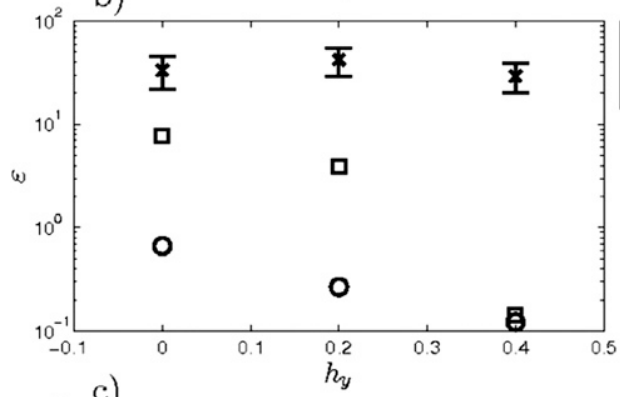

O $h_{x}=0.0$

$$
\text { 口 } h_{x}=0.2
$$

× $h_{x}=0.4$

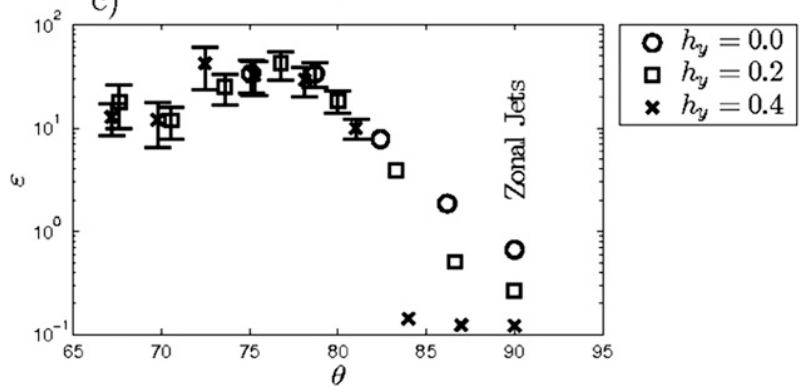

FIG. 9. Time-mean eddy energy production $\varepsilon$ as defined in section $3 \mathrm{c}$, after statistical equilibrium is reached for all two-layer runs with $\beta$ fixed at 0.75 , displayed in (a) against $h_{x}$, in (b) against $h_{y}$, and in (c) against $\theta_{\mathrm{BT}}$, the angle between $\mathbf{G}_{\mathrm{BT}}$ and $\mathbf{S}$, as defined in Eq. (16). The error bars show the standard deviation over the time that the mean was taken, shown only for points where this is bigger than the marker shown

one measure of the PV advection by the jets. There is, of course, also PV advection by the jets in the lower layer, but in the lower layer (i) the PV gradient is weaker, (ii) $q_{2}$ is not materially conserved because of bottom friction, and (iii) there is no imposed mean flow as in the upper layer. Thus upper-layer dynamics have stronger control over the drift. A plot of the jet drift in the $y$ direction $v_{\text {jet }}$ against the jet angle $\phi_{\text {jet }}$ is shown in Fig. 10 , showing an increase in the drift with increasing jet tilt. However, in this case $v_{\text {jet }}$ also depends on other measures of the flow. Indeed, we have not found an accurate scaling prediction for the magnitude of the drift produced in either one- or two-layer cases, and we will continue to investigate this in future work.

We have highlighted in this section that jet drift is a manifestation of PV conservation. The nature of the

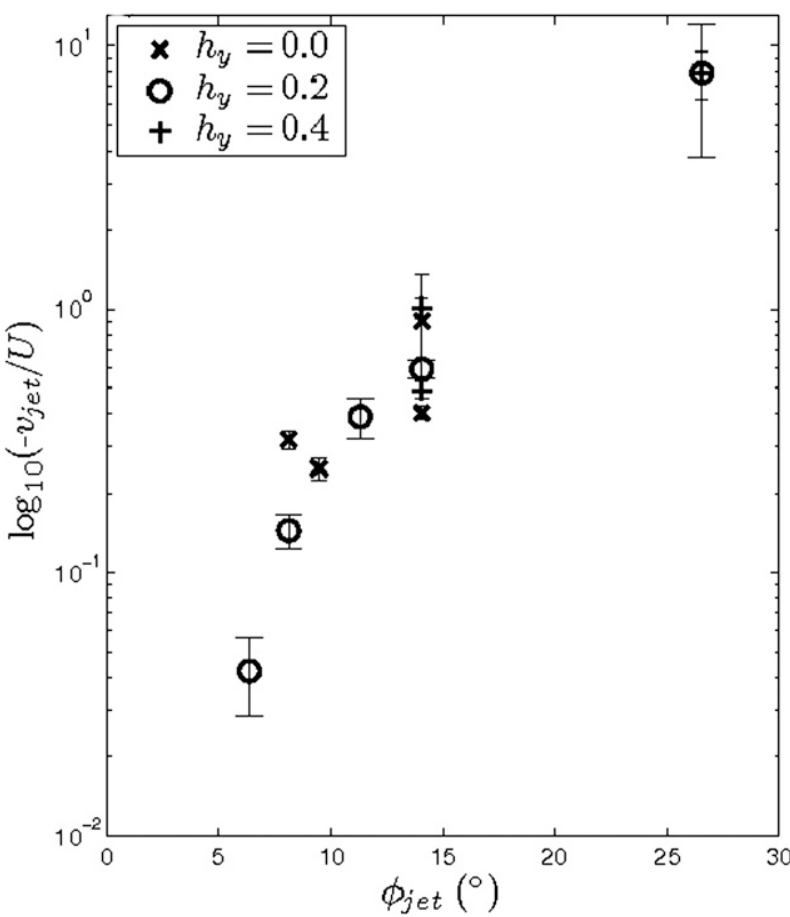

FIG. 10. Nondimensionalized jet drift in the $y$ direction $v_{\text {jet }}$ for two-layer simulations, plotted against the jet angle $\phi_{\text {jet }}$ (which is the angle between the jet and the direction orthogonal to largescale PV gradients in the upper layer). Note the log scale on the $y$ axis. All quantities are averages over time after statistical equilibrium, and the error-bars represent the standard deviation over time.

periodic domain in this study means that generation of relative vorticity is insufficient to counter advection across layer-wise PV gradients over long periods of time. We note, however, that in realistic flows, jet displacement and the subsequent modification to the planetary vorticity is a potential mechanism for conserving PV in strongly steered flows (see Thompson and Richards (2011)).

\section{e. Transport}

While the PV histograms give an idea of the transport properties of the two-layer simulations, to analyze them more quantitatively we employ the effective diffusivity diagnostic $\kappa_{\text {eff }}$ as developed by Shuckburgh and Haynes (2003), based on work by Nakamura (1996). Here, $\kappa_{\text {eff }}$ provides a measure of the relative mixing by considering the complexity of a tracer contour. Transport barriers are associated with regions of low mixing, therefore simple tracer contours and low values of $\kappa_{\text {eff }}$. Conversely, high values of $\kappa_{\text {eff }}$ are associated with regions of strong mixing. To use this diagnostic, we include a conserved passive tracer with an imposed large-scale gradient $\mathbf{g}$, so that the tracer concentration $\chi^{\mathbf{g}}$ in the $i$ th 
layer may be written in the form $\chi_{i}^{\mathbf{g}}=\chi_{i}^{\mathbf{g}^{\prime}}+\mathbf{g}_{i} \cdot \mathbf{x}$, where $\chi_{i}^{\mathbf{g}^{\prime}}$ is doubly periodic:

$$
\begin{aligned}
\frac{\partial \chi_{i}^{\mathbf{g}}}{\partial t}+J\left(\Psi_{i}, \chi_{i}^{\mathbf{g}}\right) & =d, \quad i=1,2, \quad \text { and } \\
\frac{\partial \chi_{i}^{\mathbf{g}^{\prime}}}{\partial t}+J\left(\Psi_{i}, \chi_{i}^{\mathbf{g}^{\prime}}\right) & =d+\mathbf{g}_{i} \cdot \mathbf{u},
\end{aligned}
$$

where $\mathbf{g}_{i}=\left(a_{i}, b_{i}\right), \mathbf{x}=(x, y), \mathbf{u}=\left(-\Psi_{y}, \Psi_{x}\right)$, and $\mathrm{d}$ is small-scale dissipation, applied as in (3). (This is analogous to the equations for PV constrained by $\beta$, and could be achieved in practice by considering a very large rectangular domain, the major part of which was filled with many copies of the flow represented by the doubly periodic simulation. The tracer concentration would be imposed at the boundaries of the domain to be consistent with the large scale gradient g.) By choosing either $a_{i}=0$ or $b_{i}=0$, it is possible to get a measure of the extent of meridional or zonal transport respectively.

We calculate the nondimensional ratio:

$$
\kappa_{\mathrm{eff}}\left(y_{e}, t\right)=\frac{L_{\mathrm{eq}}^{2}\left(y_{e}, t\right)}{L_{\mathrm{min}}^{2}},
$$

where $L_{\mathrm{eq}}$ is the equivalent length of a stirred contour, $L_{\min }$ is the minimum contour length, equal to the domain width for purely zonal jets, and $y_{e}$ is the equivalent latitude, defined as the latitude a given contour would have were it to be remapped to be zonally symmetric while retaining its internal area. See Shuckburgh and Haynes (2003) for full details of the calculations of these quantities. Note that $\kappa_{\text {eff }}$ is not a true diffusivity as it is dimensionless: the true effective diffusivity also depends on the numerical diffusion of the tracer. However, Shuckburgh and Haynes (2003) show that the true effective diffusivity is largely independent of the tracer numerical diffusivity.

The evolution equation for the tracers $\chi_{i},(18)$, is linear in $\chi_{i}$, and so once initial conditions have been forgotten (i.e., the tracer distribution has become independent of the initial distribution), $\chi_{i}$ are linear functions of the background gradient $\mathbf{g}$, so that

$$
\chi_{i}^{p \mathbf{g}+q \mathbf{h}}=p \chi_{i}^{\mathbf{g}}+q \chi_{i}^{\mathbf{h}} .
$$

Therefore, if $\chi_{i}^{(1,0)}$ and $\chi_{i}^{(0,1)}$ are evaluated by taking $\mathbf{g}=$ $(1,0)$ and $\mathbf{h}=(0,1)$ respectively, then $\chi_{i}^{\mathbf{g}}$ can be deduced for a general $\mathbf{g}=(a, b)$ as

$$
\chi_{i}^{\mathbf{g}}=a \chi_{i}^{(1,0)}+b \chi_{i}^{(0,1)} .
$$
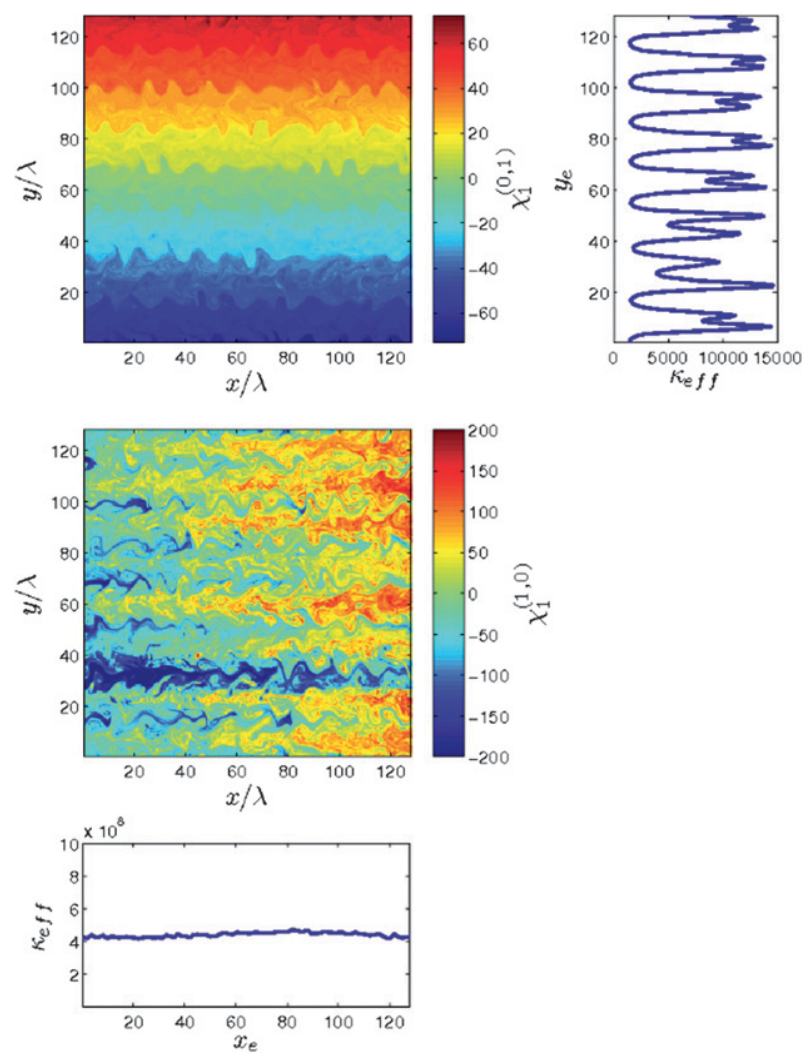

FIG. 11. Snapshots of the tracer fields (top) $\chi_{1}^{(0,1)}$ and (middle) $\chi_{1}^{(1,0)}$, for a simulation with $h_{x}=h_{y}=0$ and $\beta=0.75$, sometime after statistical equilibrium. The effective diffusivities $\kappa_{\text {eff }}$ averaged over statistical fluctuations, for (top right) $\chi_{1}^{(0,1)}$ and (bottom ) $\chi_{1}^{(1,0)}$ are shown against the equivalent latitude $y_{e}$ and the equivalent longitude $x_{e}$.

The tracer fields $\chi_{i}^{(1,0)}$ experience a zonal background gradient, and the tracer fields $\chi_{i}^{(0,1)}$ experience a meridional background gradient, and so we gain information about zonal transport from $\chi_{i}^{(1,0)}$ and information about meridional transport from $\chi_{i}^{(0,1)}$. If we look at fields with $\mathbf{g}=(a, b)=(\sin \alpha, \cos \alpha)$, where $0 \leq \alpha \leq 90$, then the fields $\sin \alpha \chi_{i}^{(1,0)}+\cos \alpha \chi_{i}^{(0,1)}$ are equivalent to the fields simulated with a tracer gradient in the direction $\alpha$. Thus, we can gain information about transport properties in a range of directions without having to run separate simulations for each value of $\alpha$ we are interested in.

We begin by testing this concept for a case with purely zonal jets, that is, $h_{x}=0$, which are known from various studies to be good transport barriers in the meridional direction and bad transport barriers in the zonal direction. Snapshots of the full tracer fields in the upper layer, $\chi_{1}^{(0,1)}$ and $\chi_{1}^{(1,0)}$, can be seen in Fig. 11 alongside the calculated $\kappa_{\text {eff }}$ plotted against $y_{e}$ and on the relevant tracer contours. It is immediately apparent that the zonal jets are excellent barriers to meridional transport, 

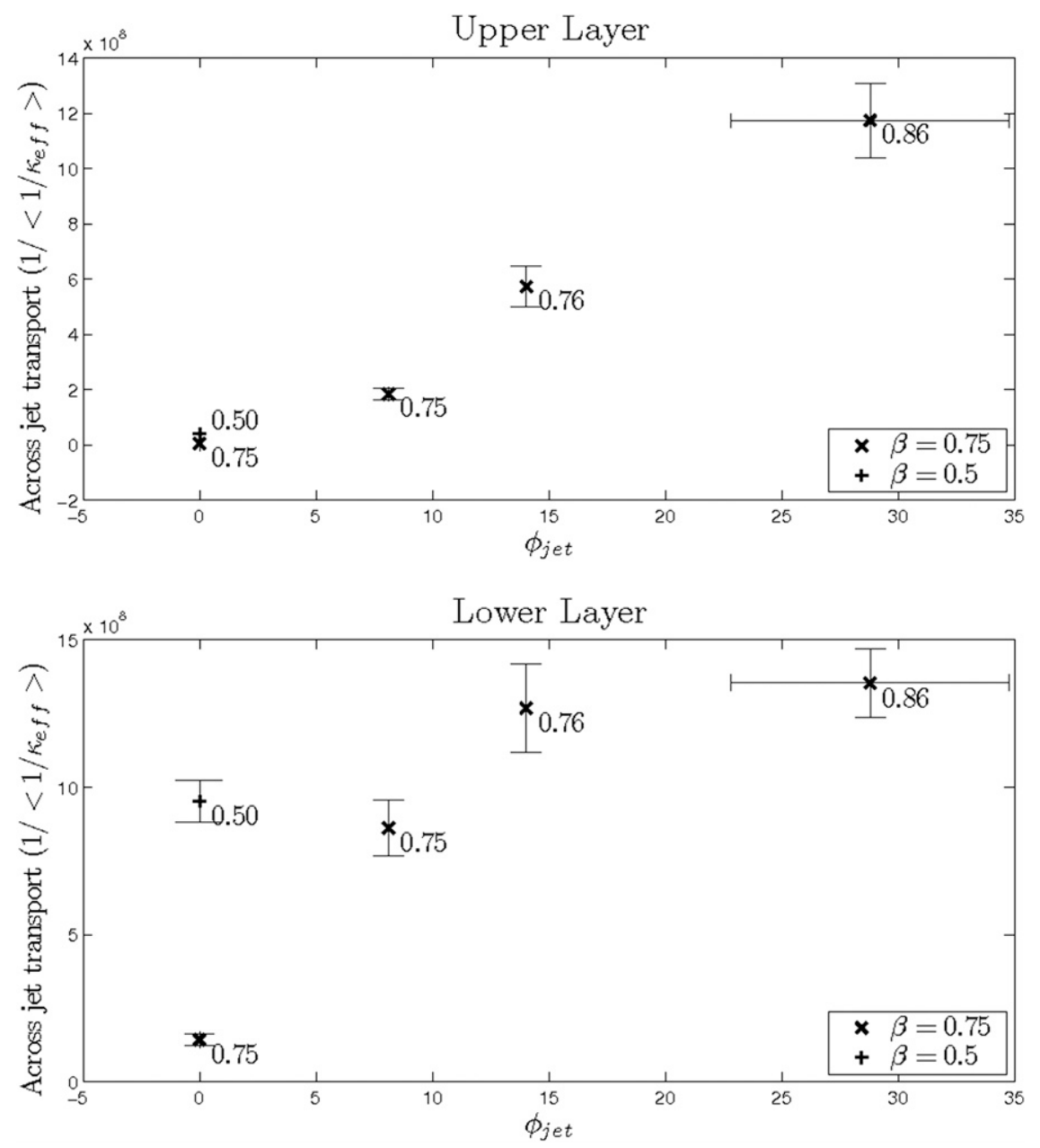

FIG. 12. Comparison of across-jet transport properties for simulations with various barotropic PV gradients $\left(\left|\mathbf{G}_{\mathrm{BT}}\right|\right.$, the label on each point $)$, and jet angles $\left(\phi_{\text {jet }}\right)$. The across-jet transport is the harmonic mean of the effective diffusivity, $1 /\left\langle 1 / \kappa_{\text {eff }}\right\rangle$, calculated from the fields $\chi_{i}^{\mathbf{g}=\left(\sin \phi_{\mathrm{jet}}, \cos \phi_{\mathrm{jet}}\right)}$, see (21). The error bars are the standard deviation over the time after statistical equilibrium. It is clear that the more tilted the jet, the greater the across-jet transport, despite increasing barotropic PV gradient magnitude.

and excellent mixers in the zonal direction. The mixing of a tracer with a zonal gradient $\chi_{1}^{(1,0)}$ by zonal jets can be directly compared to the shear dispersion of PV noted by Smith (2007). Note that $y_{e}$, the equivalent latitude, is an area coordinate, and so does not necessarily increase in the $y$-direction, as may be implied. In the case of $\chi_{1}^{(0,1)}$, the area contained by contours of successively larger tracer values does indeed increase in the positive $y$ direction. However, in the case of $\chi_{1}^{(1,0)}$, it makes more sense to think of an equivalent longitude $x_{e}$, and so we have plotted the $\kappa_{\text {eff }}$ against $x_{e}$ in this case in Fig. 11 to avoid confusion, although it is the same quantity in both cases.

Using the approach described above, it is possible to calculate $\kappa_{\text {eff }}$ in both upper and lower layers for any value of $\alpha$. We can compare the across-jet transport of runs with different measured jet deflection angles $\phi_{\text {jet }}$ by calculating the harmonic mean of the effective diffusivity, $1 /\left\langle 1 / \kappa_{\text {eff }}\right\rangle$ (shown to be proportional to the flux of tracer in, for example, Nakamura 2008) from the tracer field generated using $\alpha=\phi_{\text {jet }}$. The angular brackets here represent a mean over equivalent latitude, $y_{e}$, and time (after statistical equilibrium has been reached). Thus, we can ascertain whether the nonzonal jets are as effective barriers to across-jet transport as zonal jets.

Figure 12 shows the values of across-jet transport for various simulations with different magnitudes of barotropic PV gradient $\left(\left|\mathbf{G}_{\mathrm{BT}}\right|\right)$ and different measured deflection angles $\left(\phi_{\text {jet }}\right)$ in both the upper and lower layers. With $\phi_{\text {jet }}$ held fixed, an increase in $\left|\mathbf{G}_{\mathrm{BT}}\right|$ results in a 
decrease in across-jet transport, because of the strengthening of the jets (this is more pronounced in the lowerlayer transport where the jets are relatively weaker). However, there is a clear increase in across-jet transport with increasing $\phi_{\text {jet }}$, despite increasing $\left|\mathbf{G}_{\mathrm{BT}}\right|$. We postulate that this is because the more tilted the jets, the more they cross the layer-wise PV gradient and so the more they mix. The pattern is clearer in the upper layer, as the layer-wise PV gradient is stronger here. Overall, it is clear that tilted jets are weaker barriers to transport than their zonal counterparts, and that this tilt has a stronger effect than the change in the magnitude of the driving PV gradient, $\left|\mathbf{G}_{\mathrm{BT}}\right|$.

Comparisons of the transport for a range of values of $\alpha$ were undertaken for a variety of runs. It was expected that $\alpha=\phi_{\text {jet }}$ would give the lowest overall transport, as this represents across-jet transport. However, while $\alpha=\phi_{\text {jet }}$ produced recognizable jet and mixing regions, as is seen in the upper right panel of Fig. 11, the overall minimum transport, measured by the harmonic mean of the effective diffusivity, was found at values for $\alpha<\phi_{\text {jet }}$, where the effective diffusivity did not show clearly the jets visible in the original tracer fields. We postulate that this mismatch between the minimum transport direction and the across-jet direction is related to the jet drift discussed in section $\mathrm{d}$, although analysis is ongoing.

\section{Conclusions}

We have investigated the introduction of arbitrarily orientated linear slopes in bottom topography to simple barotropic and baroclinic quasigeostrophic models. The resulting structures have been analyzed for their transport properties and the production of energy. The most significant finding is that the formation of coherent jets, familiar from many studies without topography and also with topographic slope in the meridional direction, persists under the addition of a topographic slope in the zonal direction. The corresponding jets tilt relative to the zonal direction and cross layer-wise PV gradients, with significant implications for transport of layer-wise PV. Investigation of length and velocity scales show that the tilted jets follow Rhines scaling in both the one- and two-layer cases, although different velocity scales are used in each case, see section $3 b$.

In the two-layer case, jets follow the barotropic PV gradient, the mean of the two layer PV gradients. Analysis of the total barotropic PV through histograms confirmed that the jets formed were barriers to barotropic PV, although mixing layer-wise PV. This motivates the interpretation of the two-layer case as a single barotropic field driven by the baroclinic instability generated by the shear between the two layers.

This has interesting implications for mixing in that it implies that in regions with large-scale bottom topography, it is possible that the jet direction and layer-wise PV gradients decouple. That is, the alignment and so mixing properties of jets near the surface are determined by the direction of the barotropic PV gradient. Of course, the model presented is a highly idealized system; however, it is its very simplicity that provides further scope for testing these ideas.

Analysis of the eddy energy production $\varepsilon$ in the twolayer case shows an increase in $\varepsilon$ with decreasing angle between the background PV gradient $\mathbf{G}_{\mathrm{BT}}$ and the zonal direction. This implies a maximum of eddy energy production when the barotropic $\mathrm{PV}$ gradient $\mathbf{G}_{\mathrm{BT}}$ is aligned with the shear $\mathbf{S}$ and a minimum when $\mathbf{G}_{\mathrm{BT}}$ is at right angles with $\mathbf{S}$-see Fig. 1. These findings are consistent with those of Smith (2007), who finds large values of $\varepsilon$ with $\mathbf{G}_{\mathrm{BT}}$ and $\mathbf{S}$ aligned, and Arbic and Flierl (2004b), who find a maximum in $\varepsilon$ with $\mathbf{G}_{\mathrm{BT}}$ and $\mathbf{S}$ antialigned and a minimum with $\mathbf{G}_{\mathrm{BT}}$ and $\mathbf{S}$ at right angles.

Observations that the tilted jet simulations exhibited "jet drift" led to the hypothesis that between-jet particles in well-mixed regions are constantly transported up (down) the layer-wise PV gradients in both the oneand two-layer simulations and thus gain (lose) PV. This necessitates the jet drift that counteracts this gain (loss) in order for the system to reach statistical equilibrium. This finding highlights the importance of PV conservation, and in more realistic flow regimes, generation of relative vorticity as well as jet displacement may act to generate jet variability.

Finally, a comparison of the transport properties of zonal and nonzonal jets in the two-layer model was undertaken using an effective diffusivity diagnostic. A comparison of across-jet transport showed that jets that cross layer-wise PV gradients are weaker barriers to transport than zonal jets, which do not. This can also be thought of similarly to the simulations of Smith (2007), which show that meridional jets (extreme versions of our tilted jets) are subject to dispersion of the nonzonal shear (equivalent to our tilted barotropic PV gradient) by the beta effect, increasing cross-flow mixing. However, note that here we have set up a mechanism for shear dispersion that is internally consistent as our driving background shear is a solution to the quasigeostrophic equations.

The model presented adds to the work of those such as Thompson (2010), Wolff et al. (1991), and Witter and Chelton (1998) in furthering the understanding of topography feedback within simplified models. These models have proved to be useful guides to understanding 
dynamics in more complex and realistic circulation models, for instance in the Southern Ocean. Our study points to the role topography may play in allowing a mean flow to develop that is not orthogonal to (layer wise) mean, or background, PV gradients, which may be of importance in the ocean. However, as this study considers a broad and uniform slope, we would not seek to apply these conclusions directly to any specific part of the ocean. In particular, the quasigeostrophic assumption requires that variations in the topography are small compared to the layer depths, which limits the magnitude of slopes that can be realistically represented by this model. Alignment of the jets along the barotropic PV gradient may be sensitive to the strength of the topographic slope and the layer thicknesses. As it is well known that QG often provides reasonable results well outside of formal QG scalings, we have briefly explored simulations with values of $h_{x}$ and $h_{y}$ one and two orders of magnitude larger than the simulations discussed here. Overall the simulations produce similar results, in particular the jet alignment continues to be controlled by the barotropic PV gradient $h_{x} /\left(2 \beta+h_{y}\right)$. We note here that in this case the topographic slope dominates over the PV gradient contributions from both $\beta$ and the mean vertical shear, such that the lower-layer PV gradient is much greater than that in the upper layer and so the lower-layer jets are much stronger than those in the upper layer, and the barotropic PV gradient is very close to the lower-layer PV gradient. It would be useful to explore this steep topography regime further in a primitive equation framework.

In future work we would like to focus on theoretical explanations for the barotropic control observed in this study. In particular, we are exploring the extent to which linear Rossby wave theory could apply. This relates to the theory, first introduced by Rhines (1975) and much expanded by Vallis and Maltrud (1993), that zonal jets form in the presence of $\beta$ due to the arrest of the turbulent energy cascade when Rossby waves are efficiently excited. The anisotropy of the Rossby wave dispersion relation causes the preferred direction to be the zonal one. Hallberg (1997) clearly shows how this dispersion relation is altered when arbitrarily orientated PV fields are allowed in the freely decaying case, showing a rotation of the classic dumbbell shape. It would be interesting to see how the behavior changes in this forced-dissipative case, and the dependence on the strength of the topographic slope.

There are also many possible ways to develop the current models to make them more relevant to localized topographical regions in the Southern Ocean, including using a different ratio of layer depths to better mimic stratification in the Southern Ocean. We would be interested to discover if the barotropic behavior held in multiple-layer systems with more realistic stratifications and topographies. We also plan to motivate any further development of the model through a combination of modeling and data analysis of topographically complex regions of the Southern Ocean.

Acknowledgments. We thank two anonymous reviewers for their helpful and interesting comments. We would like to acknowledge Samuel Bouvier for conducting some simulations at the start of this project. AFT was supported by a NERC Advanced Research Fellowship (NE/H015760/1). EJDB was supported by the international DIMES project.

\section{REFERENCES}

Abernathey, R., J. Marshall, M. Mazloff, and E. Shuckburgh, 2010: Enhancement of mesoscale eddy stirring at steering levels in the Southern Ocean. J. Phys. Oceanogr., 40, 170-184.

Arbic, B. K., and G. R. Flierl, 2004a: Baroclinically unstable geostrophic turbulence in the limits of strong and weak bottom Ekman friction: Application to midocean eddies. J. Phys. Oceanogr., 34, 2257-2273.

—_, and ——, 2004b: Effects of mean flow direction on energy, isotropy, and coherence of baroclinically unstable betaplane geostrophic turbulence. J. Phys. Oceanogr., 34, 77-93.

Arnold, V. I., and L. D. Meshalkin, 1960: A.N. Kolmogorov's seminar on selected problems of analysis (1958/1959). Uspekhi Mat. Nauk, 15, 247-250.

Bastin, M., and P. Read, 1998: Experiments on the structure of baroclinic waves and zonal jets in an internally heated, rotating, cylinder of fluid. Phys. Fluids, 10, 374-389.

Berloff, P., I. Kamenkovich, and J. Pedlosky, 2009: A model of multiple zonal jets in the oceans: Dynamical and kinematical analysis. J. Phys. Oceanogr., 39, 2711-2734.

Beron-Vera, F. J., M. G. Brown, M. J. Olascoaga, I. I. Rypina, H. Kocak, and I. A. Udovydchenkov, 2008: Zonal jets as transport barriers in planetary atmospheres. J. Atmos. Sci., 65, 3316-3326.

Gordon, A. L., E. Molinelli, and T. Baker, 1978: Large-scale relative dynamic topography of the Southern Ocean. J. Geophys. Res., 83 (C6), 3023-3032.

Haidvogel, D. B., and I. M. Held, 1980: Homogeneous quasigeostrophic turbulence driven by a uniform temperature gradient. J. Atmos. Sci., 37, 2644-2660.

Hallberg, R., 1997: Localized coupling between surface and bottom-intensified flow over topography. J. Phys. Oceanogr., 27, 977-998.

—, and A. Gnanadesikan, 2006: The role of eddies in determining the structure and response of the wind-driven southern hemisphere overturning: Results from the Modeling Eddies in the Southern Ocean (MESO) project. J. Phys. Oceanogr., 36, 2232-2252.

Hart, J. E., 1975: Baroclinic instability over a slope. Part I: Linear theory. J. Phys. Oceanogr., 5, 625-633.

Held, I. M., and V. D. Larichev, 1996: A scaling theory for horizontally homogenous, baroclinically unstable flow. J. Atmos. Sci., 53, 946-952. 
Hughes, C. W., 2005: Nonlinear vorticity balance of the Antarctic Circumpolar Current. J. Geophys. Res., 110, C11008, doi:10.1029/ 2004JC002753.

—- and E. R. Ash, 2001: Eddy forcing of the mean flow in the Southern Ocean. J. Geophys. Res., 106 (C2), 2713 2722.

Jackson, L., C. W. Hughes, and R. G. Williams, 2006: Topographic control of basin and channel flows: The role of bottom pressure torques and friction. J. Phys. Oceanogr., 36, 1786-1805.

LaCasce, J. H., and K. H. Brink, 2000: Geostrophic turbulence over a slope. J. Phys. Oceanogr., 30, 1305-1324.

Lee, M. M., and A. Coward, 2003: Eddy mass transport for the Southern Ocean in an eddy-permitting global ocean model. Ocean Modell., 5, 249-266.

Lu, J., and K. Speer, 2010: Topography, jets, and eddy mixing in the Southern Ocean. J. Mar. Res., 68, 479-503.

Marshall, D., 1995: Topographic steering of the Antarctic Circumpolar Current. J. Phys. Oceanogr., 25, 1636-1650.

Marshall, J., D. Olbers, H. Ross, and D. Wolfgladrow, 1993: Potential vorticity constraints on the dynamics and hydrography of the Southern Ocean. J. Phys. Oceanogr., 23, 465-487.

_ , E. Shuckburgh, H. Jones, and C. Hill, 2006: Estimates and implications of surface eddy diffusivity in the Southern Ocean derived from tracer transport. J. Phys. Oceanogr., 36, 18061821

Mason, P., 1975: Baroclinic waves in a container with sloping end walls. Proc. Roy. Soc., A278, 397-445.

Maximenko, N., B. Bang, and H. Sasaki, 2005: Observational evidence of alternating zonal jets in the world ocean. Geophys. Res. Lett., 32, L12607, doi:10.1029/2005GL022728.

Nakamura, N., 1996: Two-dimensional mixing, edge formation, and permeability diagnosed in an area coordinate. J. Atmos. Sci., 53, 1524-1537.

_ 2008: Sensitivity of global mixing and fluxes to isolated transport barriers. J. Atmos. Sci., 65, 3800-3818.

Panetta, R. L., 1993: Zonal jets in wide baroclinically unstable regions-Persistence and scale selection. J. Atmos. Sci., 50, 2073-2106.

Rhines, P., 1975: Waves and turbulence on a beta-plane. J. Fluid Mech., 69, 417-443.

Samelson, R., 1992: Surface-intensified Rossby waves over rough topography. J. Mar. Res., 50, 367-384.

Shuckburgh, E., and P. Haynes, 2003: Diagnosing transport and mixing using a tracer-based coordinate system. Phys. Fluids, 15, 3342-3357.

— - H. Jones, J. Marshall, and C. Hill, 2009a: Robustness of an effective diffusivity diagnostic in oceanic flows. J. Phys. Oceanogr., 39, 1993-2009.

$-, \ldots, \ldots$, and,$- 2009 \mathrm{~b}$ : Understanding the regional variability of eddy diffusivity in the Pacific Sector of the Southern Ocean. J. Phys. Oceanogr., 39, 2011-2023.

Smith, K. S., 2007: Eddy amplitudes in baroclinic turbulence driven by nonzonal mean flow: Shear dispersion of potential vorticity. J. Phys. Oceanogr., 37, 1037-1050.
G. Boccaletti, C. C. Henning, I. Marinov, C. Y. Tam, I. M. Held, and G. K. Vallis, 2002: Turbulent diffusion in the geostrophic inverse cascade. J. Fluid Mech., 469, 13-48.

Sokolov, S., and S. R. Rintoul, 2007: Multiple jets of the Antarctic circumpolar current South of Australia. J. Phys. Oceanogr., 37, 1394-1412.

Spall, M. A., 2000: Generation of strong mesoscale eddies by weak ocean gyres. J. Mar. Res., 58, 97-116.

Steinsaltz, D., 1987: Instability of baroclinic waves with bottom slope. J. Phys. Oceanogr., 17, 2343-2350.

Straub, D., 1994: Dispersive effects of zonally varying topography on quasigeostrophic Rossby waves. Geophys. Astrophys. Fluid Dyn., 75 (2-4), 107-130.

Tailleux, R., and J. McWilliams, 2000: Acceleration, creation, and depletion of wind-driven, baroclinic Rossby waves over an ocean ridge. J. Phys. Oceanogr., 30, 2186-2213.

Tamaki, K., and K. Ukaji, 2003: An experimental study of wave dispersion in a differentially heated rotating fluid annulus with a radially sloping bottom. J. Meteor. Soc. Japan, 81, 951-962.

Thompson, A. F., 2010: Jet formation and evolution in baroclinic turbulence with simple topography. J. Phys. Oceanogr., 40, $257-278$

—, and W. R. Young, 2007: Two-layer baroclinic eddy heat fluxes: Zonal flows and energy balance. J. Atmos. Sci., 64, 3214-3231.

- and K. J. Richards, 2011: Low frequency variability of southern ocean jets. J. Geophys. Res., 116, C09022, doi:10.1029/ 2010JC006749.

— , P. H. Haynes, C. Wilson, and K. J. Richards, 2010: Rapid Southern Ocean front transitions in an eddy-resolving ocean GCM. Geophys. Res. Lett., 37, L23602, doi:10.1029/2010GL045386.

Treguier, A. M., and J. C. McWilliams, 1990: Topographic influences on wind-driven, stratified flow in a beta-plane channel-An idealized model for the Antarctic Circumpolar Current. J. Phys. Oceanogr., 20, 321-343.

_ , and R. L. Panetta, 1994: Multiple zonal jets in a quasigeostrophic model of the Antarctic Circumpolar Current. J. Phys. Oceanogr., 24, 2263-2277.

Vallis, G. K., and M. E. Maltrud, 1993: Generation of mean flows and jets on a beta plane and over topography. J. Phys. Oceanogr., 23, 1346-1362.

Whitehead, J., M. Stern, G. Flierl, and B. Klinger, 1990: Experimental observations of baroclinic eddies on a sloping bottom. J. Geophys. Res., 95 (C6), 9585-9610.

Witter, D. L., and D. B. Chelton, 1998: Eddy-mean flow interaction in zonal oceanic jet flow along zonal ridge topography. J. Phys. Oceanogr., 28, 2019-2039.

Wolff, J. O., E. Maierreimer, and D. J. Olbers, 1991: Wind-driven flow over topography in a zonal beta-plane channel-A quasigeostrophic Model of the Antarctic Circumpolar Current. J. Phys. Oceanogr., 21, 236-264.

Wordsworth, R. D., P. L. Read, and Y. H. Yamazaki, 2008: Turbulence, waves, and jets in a differentially heated rotating annulus experiment. Phys. Fluids, 20, 126602, doi:10.1063/ 1.2990042 